Lubrich, O. - Sprachbilder des Krieges

\title{
Sprachbilder des Krieges. Zur ersten Fassung von Ernst Jüngers In Stahlgewittern
}

\author{
Oliver Lubrich ${ }^{1}$
}

\begin{abstract}
In his controversial first book In Stahlgewittern (1920) Ernst Jünger ascribes meaning to World War I not explicitly in ideological terms, as much as indirectly by using a highly metaphorical language. Jünger codes war in 32 image sequences in the fields of nature, human practice, culture, and anthropomorphism. Jünger's metaphors can be differentiated into various categories and characterized by their density, their relation, their interference and their variation. When two warring fractions, then, are described by the same iconic motifs, their oppositions seem to vanish. And when metaphors are confronted with their real referents, with signifying names and stereotyped jargons, their artificial character and epistemological function come to light. The implicit meanings these metaphors generate should not be simplified as celebratory aestheticising, nor refuted as fascist ideology. The individual codes are unequally subtle, they generate different semantics and they connote deviating political positions; they intersect and conflict. In Stahlgewittern they are both an expression of an ideological attitude as well as a symptom of profound unsettlement.
\end{abstract}

Keywords: Ernst Jünger; War; Metaphor; Ideology

Zusammenfassung: In seinem umstrittenen Erstlingswerk, In Stahlgewittern (1920), schreibt Ernst Jünger dem Ersten Weltkrieg weniger explizit einen ideologischen Sinn zu, als er ihn indirekt durch eine intensiv metaphorische Sprache mit Bedeutungen auflädt. Jünger codiert den Krieg in 32 bildlichen Sequenzen, die sich in den Feldern der Natur, der menschlichen Praxis, der Kultur und der Anthropomorphie verorten. Diese Sprachbilder lassen sich nach Typen differenzieren und in ihrer Dichte, Relation, Interferenz und Variation beschreiben. Dadurch dass dieselben Bilder auf beide Kriegsparteien angewandt werden, relativieren sie deren Gegensatz. Indem die Metaphern mit ihren realen Entsprechungen, mit sprechenden Namen und mit stereotypen Jargons konfrontiert werden, tritt ihre Künstlichkeit zutage und wird ihre epistemische Funktion deutlich. Die implizite Bedeutungs-Zuschreibung, die diese Metaphoriken erzielen, ist keineswegs einsinnig als Ästhetisierung oder Verherrlichung bzw. als kohärente faschistische Ideologie zu fassen. Die einzelnen Codes sind ungleichmäßig subtil, sie erzeugen verschiedene Bedeutungen, und sie konnotieren abweichende politische Positionen; sie geraten miteinander in Überschneidung und zueinander in Widerspruch. Es entsteht eine widerständige Semantik, die als Symptom einer Verunsicherung lesbar ist.

\footnotetext{
1 Juniorprofessor für Rhetorik am Peter Szondi-Institut für Allgemeine und Vergleichende Literaturwissenschaft sowie am Exzellenzcluster „Languages of Emotion“ der Freien Universität Berlin. Email: lubrich@zedat.fu-berlin.de.
} 


\section{Lubrich, O. - Sprachbilder des Krieges}

Stichwörter: Ernst Jünger; Krieg; Metapher; Ideologien

Ernst Jüngers In Stahlgewittern $(1920)^{2}$ enthält kaum explizite Reflexionen über den Ersten Weltkrieg. Zwei Dutzend direkten Aussagen stehen einhundert intertextuelle Referenzen und vor allem viele Hundert Sprachbilder gegenüber, die zahlreiche Bedeutungen erzeugen. Inwiefern diese bildliche Codierung das vorherrschende Verfahren der Kriegsdeutung ist, lässt sich jedoch nicht allein quantitativ ermessen. Im Folgenden soll der Versuch dokumentiert werden, Jüngers Bildsprache struktural zu analysieren und ihre implizite Semantik zu entziffern. Wie inszeniert Jünger den Krieg metaphorisch? Und welche Sinnzuschreibungen werden über seine Bilder vermittelt?

\section{Metaphorik}

Auf den ersten Seiten legt Jünger die Spuren, denen seine Leser durch den Text hindurch folgen können, indem er in großer Dichte eindrückliche Sprachbilder plaziert. ${ }^{3}$ Durch ein bildliches Vokabular der Beschreibung erhält der Text eine zusätzliche Ebene, auf der sein Gegenstand modellhaft figuriert. Im Metaphorischen, so scheint es, lässt sich das Unbegreifliche am ehesten in vertraute Zusammenhänge bringen (Vgl. BLUMENBERG 1960; 1981; LAKOFF/JOHNSON 1980). Jünger übersetzt seine Erfahrung

\footnotetext{
2 Als Textgrundlage dient die Erstausgabe von 1920, die erste von sieben ,Fassungen', zwischen denen der Autor erhebliche Überarbeitungen vorgenommen hat: Ernst JÜNGER, In Stahlgewittern. Aus dem Tagebuch eines Stoßtruppführers. Hannover, 1920. (Für alle Zitate nach dieser Ausgabe werden im Text die Seitenzahlen in Klammern angegeben.) Die weiteren sechs ,Fassungen“ erschienen 1922, 1924, 1934, 1935, 1961 und 1978: In Stahlgewittern. Aus dem Tagebuch eines Stoßtruppführers. Berlin, 1922 (2. Fassung).- Berlin, 1924 (3. Fassung).- In Stahlgewittern. Ein Kriegstagebuch. Berlin, 1934 (4. Fassung).- Berlin, 1935 (5. Fassung).- In Stahlgewittern. In: Werke. Stuttgart, 1961, Band 1, S. 11-310 (6. Fassung).- In: Sämtliche Werke 1, S. 9-300; separat publiziert: Stuttgart, $1981^{27}$ (7. Fassung). Hermann KNEBEL (1991) untersuchte die quantitativ und qualitativ erheblichen Veränderungen. Knebel geht davon aus, dass Jünger ein „generatives Zentrum“ (das von Harro Segeberg herausgearbeitete Denkmodell der „Regressiven Modernisierung“) in jeweils verschiedener Weise realisierte. Die Programmatik verschiebt sich dabei von der Erinnerung des Kriegsteilnehmers über die Agitation des revolutionären Nationalisten zur humanistischen Literatur des elitären Schriftstellers. - Vergleiche auch KUNICKI 1993.

${ }^{3}$ Der Untertitel, Aus dem Tagebuch eines Stoßtruppführers, bezieht den Text auf reale Aufzeichnungen, die im Krieg entstanden sind. Das Original-Tagebuch behielt Jünger bis zu seinem Tod im Privatbesitz. Es erschien erst 90 Jahre nach der Erstausgabe seiner literarischen Ausarbeitung als Kriegstagebuch
} 


\section{Lubrich, O. - Sprachbilder des Krieges}

in eine kunstvoll durchkomponierte Metaphernsprache, die semantischen Codes entspricht und alternative Interpretationen eröffnet. ${ }^{4}$ Er führt sein Thema gleichsam in unterschiedlichen Tonarten durch.

Nach Friedrich Nietzsche und Paul de Man wäre ohnehin jedes Wort mindestens insofern mehrfach lesbar, als zwischen seiner ,buchstäblichen' (vordergründigen, pragmatischen) und seiner ,rhetorischen' (auf den ersten Blick oft übersehenen) Bedeutung zu entscheiden ist (Vgl. NIETZSCHE [1873] 1988; DE MAN 1979). Sobald bestimmte Figurationen proliferieren, treten sie zueinander in ein Verhältnis wechselseitiger Bestätigung und Verdeutlichung. Einzelne Metaphern, im erweiterten, aristotelischen Sinn, ${ }^{5}$ verbinden sich zu Bedeutungsketten. Sie konstituieren einen Subtext, sobald sich mehrere zu einer Verweisstruktur zusammenfügen. In die verschiedenen kommunikativen Strategien des Textes - Beschreibung, Schilderung, Reflexion und Analyse - sind Tropen eingelagert, die aufeinander reagieren. Aus einzelnen, verstreuten Elementen entstehen Sequenzen, Bereiche, Strukturen. Diese sind nicht strikt voneinander getrennt, sondern sie berühren und überschneiden sich. Verschiedene Subtexte ergänzen einander zu umfassenden Systemen der Signifikation.

Im Wesentlichen lassen sich vier Felder unterscheiden: Jünger codiert den Krieg, erstens als Ereignis der Natur, zweitens als ökonomische Praxis, drittens als Phänomen der Kultur und viertens als Anthropomorphismus. ${ }^{6}$

1914-1918, herausgegeben von Helmuth Kiesel (Stuttgart, 2010). John KING (2003) hatte zuvor die Aufzeichnungen mit den Druckfassungen verglichen.

${ }^{4}$ Hermann Knebel macht Andeutungen hinsichtlich einer „Einbettung des Kriegsgeschehens in sinnstiftende Diskurse“, die Jünger implizit und latent ,über die Darstellungstechnik“ erzeugt (KNEBEL 1991: 391). Harro Segeberg spricht von einem ,absichtsvoll durchkomponierten Text mit beziehungsreich angeordneten Sprachbildern“ (SEGEBERG 1991: 344 f.). Die umfassendste Studie - zu Jüngers literarischen Werken bis 1933 - hat Hans VERBOVEN (2003: 15-27, methodischer Teil) vorgelegt.

${ }^{5}$ Poetik, 21-22; Rhetorik, III.2, 4, 10, 11.

${ }^{6}$ Es muss darauf verzichtet werden, im einzelnen die Belege anzuführen, deren vollständige Erhebung der folgenden modellhaften Rekonstruktion zugrunde liegt. Vergleiche LUBRICH 2003: 148-224; sowie die auf In Stahlgewittern sich beziehenden Abschnitte bei VERBOVEN 2003, der eine alternative Strukturierung vorschlägt: S. 29-35 (Wasser), S. 55-61 (Feuer), S. 81-85 (Musik), S. 96 f. (Schauspiel), S. 110-115 (Produktion), S. 148-152, S. 167 ff. und S. 175 ff. (Verwandlung), S. 182 ff. (Jagd), S. 190 f. (Rausch), S. 195 ff. (Übernatürliches), S. 203 ff. (Raum). 


\section{Lubrich, O. - Sprachbilder des Krieges}

\section{Erstes Feld: Natur}

Der Krieg figuriert als Naturereignis:

(1) Kosmos: Auf höchster Ebene lässt er sich in Bilder der Astronomie bringen: Ein großes Geschoss erscheint dann als Sonne, Explosionen im Hintergrund als Planeten und eine Kampfstellung als Mond.

(2) Wetter: Bereits im Titel, In Stahlgewittern, ist der Krieg als klimatischer Vorgang verbildlicht. Und Jünger dekliniert dieses Motiv durch: Beschuss oder Offensiven erscheinen als Unwetter, Sturm, Orkan mit Donner, Blitzen, Wolken, wirbelndem oder tosendem Wind, Hagel und Regen, in starkem Prasseln, als leichte Schauer oder sogar als Schnee. Sie kündigen sich an, indem zu spüren ist, dass „,etwas in der Luft liegen mußte“ $(35,45)$ oder dass die „Stimmung [...] schwül“ wird (37). Sie brausen auf; und flauen ab. Was zurückbleibt, ist eine Art Tau, der für menschliches Blut steht. Der Einzelne sieht sich „einem Ausbruch der Elemente gegenüber“ (51 f.). Als künstliches Naturereignis drängt sich der Krieg gegenüber der eigentlichen Natur in den Vordergrund: „Selbst die Naturgesetze“, heißt es über einen Angriff im Frühjahr, ,schienen ihre Gültigkeit verloren zu haben; die Luft flimmerte wie an heißen Sommertagen.“(144)

(3) See: In ihren Gräben fühlen sich die Soldaten wie auf einer Insel von einem Taifun umtost oder wie auf einem in Seenot geratenen Schiff. Das Schlachtfeld mit seinen Trichtern ist dann das Meer, die Unebenheiten des Geländes werden zu Wellen. Als Offizier ist Ernst Jünger der „Kapitän“ (163). Die Bewegungen seines Stoßtrupps durch die Nebel des nächtlichen Niemandslandes werden zu „Irrfahrten“ (119). In Ausformung dieser Bildlichkeit erscheinen Stellungen als Küste und Verteidigungswälle als Deiche, auf die das Meer des feindlichen Beschusses und die Wogen der Angriffe zulaufen wie eine Brandung, die sich mit der Regelmäßigkeit von Gezeiten nähert und wieder zurückzieht. Die Bewegungen der Truppen werden sichtbar als Ströme, die aus dem Hinterland der Front zufließen und sich dort aufstauen, bevor sie sich ins Meer der Todeszone ergießen und auf die feindlichen Linien zurollen. Der einzelne Soldat wird wie von einer Strömung oder einem Strudel mitgerissen. Und sogar das Leid ist in derselben Metaphorik erfasst: Das Blut scheint, wie Wasser, zu strömen. 


\section{Lubrich, O. - Sprachbilder des Krieges}

(4) Land: Alternativ zur Vorstellung von Wasser und Meer durchläuft das Kriegsgebiet eine Metamorphose verschiedener Landschaftsformen: Wüsten, Felder, Wälder, Hügel, Geisire und Vulkane. Während sich einerseits reales „Ackerland zur Wüste verwandelt“ (60), wird das Kriegsgebiet andererseits zu einem „Feld“. Die Masse der Detonationen zeigt sich als „Wald von Einschlägen“ (94). Geschosse, die im durchnässten Boden auftreffen, bringen „hochspritzende[] Schlammgeisire“ (98) hervor. Trümmer von Dörfern werden zu ,gewaltige[n] Hügel[n]“ (50). Kanonaden lassen „die Erde erzittern“ (143), wie ein Erdbeben - oder „,vulkanartige[] Explosionen“ (144). Am Ende hat die Artillerie eine neue Landschaftsform geschaffen: die „Trichterlandschaft“ (121). Die Soldaten passen sich dieser Bildlichkeit ein: Wie Pflanzen sind sie mit ihrem „Abschnitt“ im Graben „verwachsen“ (25); und „dem Gegner ein Dorn im Auge“ (80).

(5) Tiere: Der Krieg erscheint als Treiben der Fauna. Soldaten, Panzer, Flugzeuge, Geschütze und Geschosse phantasiert Jünger als Tiere. Männer werden zu Tigern. Im Rausch kämpfen sie wie „Werwölfe, die heulend durch die Nacht hetzen“ (146). Solche, die Gefallene ausplündern, verhalten sich wie „Hyänen []“ (142). Der Stoßtruppführer versteckt sich in einem „Fuchsloch“ (53). Ein Sterbender „dehnte sich fast wohlig wie eine Katze“ (158). Nach verschiedenen Kriterien inszeniert Jünger Soldaten als Hunde, Schweine, Maulwürfe, Igel, Hühner, Robben, Amphibien, Eidechsen, Schlangen oder Ameisen. Mitunter vermerkt er auch, welche Eigenschaften von denen eines Tieres fehlen: „[I]ch bin keine Eule, die ihren Weg im Dunkeln findet!“ (114) Die Tierwerdung wird dadurch deutlich, dass sich Hände in Krallen verwandeln. Menschen aktivieren ihre „niederen Instinkte[]“ (71). Bisweilen müssen sie wie Pferde ,gezügelt““ (174) werden. Beim Antreten zum Angriff fühlen sich die Kämpfer, wie ,,[w]enn ein Tier der Wildnis aus seiner Höhle hervorgezerrt wird“ (143). Wie Insekten agieren sie in Schwärmen. Wie Vögel flattern sie auseinander, oder sie richten sich ein in Nestern. Sie kämpfen, bis sie „wie ein erschöpftes Tier zusammenbr[e]ch[en]“ (102). Verwundete geben ein „Geheul“ (15) von sich, „als ob sie am Spieß stäken“ (86). Tote verwandeln sich, verwesend, in Fische. Und auch der Krieg selbst ist ein Tier: Er zeigt seine „Krallen“ (2). Die Kanonen, ihre Granaten und deren Einschläge verhalten sich wie Wölfe: Sie heulen, fauchen, bellen, brüllen und verschlingen. Mitunter erkennt man sie an ihrem vogelhaft flatternden Geräusch. Oder sie wühlen sich maulwurfsgleich in den Boden. Gewehrfeuer erzeugt den insektenhaften Eindruck eines Bienenschwarms. Handgranaten sehen aus wie Eier. Gräben und Wege nehmen die Form von Schlangen 


\section{Lubrich, O. - Sprachbilder des Krieges}

an. Tanks bewegen sich „gleich unbeholfenen Riesenkäfern“ (165). Flugzeuge, die unheilvoll über ihm kreisen, erlebt der Infanterist als Aasgeier, während er eigene Maschinen als Flamingos wahrnimmt. Das Surren der Motoren erinnert ihn an Moskitos.

\section{Zweites Feld: Praxis}

Planeten, Wetter, See, Landschaft und Tierwelt liegen außerhalb menschlichen Handelns. Eine zweite Reihe von Bildlichkeiten bringt genau dies zur Sprache: verschiedene Formen menschlicher Praxis.

(6) Jagd: Eine der frühesten Formen organisierten Handelns ist die Jagd. Jünger sieht den Kampf als Hetz- oder Treibjagd, in der sich die Rollen von Jäger und Wild vertauschen. Einzelne Aspekte lassen sich als Lauer, Pirsch oder Fallenstellen inszenieren. Menschen „sprangen wie die Hasen hin und her“ (100); wenn sie getroffen wurden, „schlugen [sie] Rad wie getroffene Hasen“ (179). „Mit der gesteigerten Freude eines Weidmannes sah ich, daß wir einen gewaltigen Fang gemacht hatten“ (129). - Er selbst, erzählt Jünger, ,jagte“ Plünderer fort (142). Immer wieder gebraucht er das Basiswort ,jagen“ auch im Hinblick auf Geschütze und Geschosse: Von berittenen Batterien schreibt er, dass sie „,vorjagten“ (126). „[Es] jagte eine Explosion die andere“ (97).

(7) Viehzucht: Aus dem Bereich der Viehzucht leiten sich drei Vorstellungen ab: die von der willenlosen Herde, aus der sich der tapfere Einzelne abhebt; des Weiteren die, dass feindliches Feuer die Truppen wie Vieh (zurück-)treibt; und schließlich die, dass Soldaten, massenhaft geschlachtet werden.

(8) Ackerbau: Diverse Sprachbilder machen den Krieg zu einem landwirtschaftlichen Vorgang. Zunächst wird das Kampfgebiet, wie gesehen, von einer natürlichen Landschaft zu einem bearbeiteten Feld. Die Armeen bewirtschaften es, indem sie Anhebungen beseitigen, mit ihren Geschossen furchen, pflügen, umwühlen, umwälzen und wie mit der Hacke bearbeiten. Dann pflanzen und säen sie. Als Gegenstand dieser Landwirtschaft werden die Soldaten phantasiert: Sobald sie in Blüte stehen, werden sie geerntet, niedergemäht. Ihre Körper gehen wie Dünger in den Kreislauf der Materie ein. Bei Kriegsende kommt beides zusammen, die Aussaat des Kampfes und die Früchte des Sieges. Zunächst hat man sich Gewehrkugeln als „Bleikerne“ (85) vorzustellen, später 


\section{Lubrich, O. - Sprachbilder des Krieges}

werden Handgranaten als ,zitronenförmig[]“ (110) beschrieben. Am Ende kann Jünger trotz der Niederlage seine „Früchte des Sieges“ (153) ernten, als man ihm im Lazarett „Zitronenlimonade“ serviert (181) und den Orden „Pour le Mérite“ verleiht (181).

(9) Handwerk: „Der Krieg ist der Handwerke härtestes“, die Soldaten sind „seine Meister“ (71). Sie bearbeiten einander mit Hammer und Amboss, mit Zange und Schrauben; sie stanzen, hobeln, bohren. Von Militärärzten heißt es, sie „walteten [...] ihres blutigen Handwerkes.“ (64) Neben dem Schlosser- und dem Tischlerberuf werden zwei Gewerbe benannt: der Müller und der Uhrmacher. Der Krieg selbst wird zu einer Mühle, in der Menschen und Dinge zermahlen werden. In beschossenen Unterständen leiden Soldaten unter dem „Uhrwerk fallender Tropfen“ (VI). Allmählich werden sie stumpf wie abgenutzte Werkzeuge.

(10) Bergbau: Der Stellungskrieg ist insofern mit dem Bergbau vergleichbar, als Gräben und Bunker wie Stollen erscheinen und die Soldaten, die sie graben, wie Bergarbeiter. In ihren metertief in die Erde getriebenen Schutzlöchern verbringen sie ihr „Leben kümmerlich unter Tage“ (V).

(11) Industrie: Indem er die Bilder ökonomischen Handelns technisiert und modernisiert, gestaltet Jünger den Krieg als industrielle Produktion. Diese Bildlichkeit funktioniert auf zwei Ebenen: Im Großen gesehen, ist das Schlachtfeld eine Fabrik: eine Art Meta-Maschine, ein gigantischer Betrieb, ein Getriebe, in dem unzählige Hebel wirksam werden und ein Rädchen ins andere greift. Innerhalb dieser Fabrik sind diverse Instrumente, Mechanismen und Prozesse am Werk: Maschinen, Apparate, Techniken, Material und Energie. Aber was ist das für eine Fabrik? Was produziert sie? Das einzige konkrete Motiv, das sich herausbildet, ist das eines Eisenwerkes, in dem aus dem Rohstoff Platten hergestellt werden: ein Walzwerk. Diese Metapher wird stark profiliert und auf diverse Aspekte angewandt: auf Panzer, Geschosse, Gas - und Truppen. Die Soldaten sind dabei nicht nur die Fabrikarbeiter, sondern auch der Rohstoff, der eingeschmolzen, mit Stahl bearbeitet und zu einem Produkt geformt wird. (Immer wieder werden sie „,verladen“, ,,abtransportiert“ und wieder ,,ausgeladen“.)

(12) Arbeit: Mit dem Begriff „Arbeit“ belegt Jünger die Tätigkeit im Schützengraben oder im Hinterland, konkret das Ausheben von Befestigungen, das Vordringen zu den feindlichen Linien, das Schießen und Töten - und das Bergen von Leichen aus Trümmern und Schlamm. Das Motiv wird allerdings nicht auf handwerkliche oder in- 


\section{Lubrich, O. - Sprachbilder des Krieges}

dustrielle Tätigkeiten beschränkt, sondern auf behördliche oder freiberufliche erweitert. Der Krieg wird zu einer Berufstätigkeit, in der Beamte und Angestellte ihre Spezialgebiete haben und nach langen „Arbeitszeiten“ ihren Feierabend genießen. (Der Begriff ist auch auf die Gegner angewandt, jedoch nur im engeren Sinn der Schanzarbeit.)

(13) Ökonomie: Auch in der Handels- und Finanzökonomie ist der Krieg als Phänomen des Kapitalismus zu verstehen. Jünger verwendet eine weite Palette wirtschaftlicher Begriffe, um einzelne Aspekte zu illustrieren: Geschäft, Geld, Kosten, Preis, Sparen, Etat, Taxe, Zoll, Export, Tausch, Vertrag, Verkauf und Quittung.

\section{Drittes Feld: Kultur}

Der Krieg wird kulturalisiert. Er wird kollektiven Modellen des Weltverständnisses eingegliedert und in der Form entsprechender kultureller Praktiken inszeniert.

(14) Religion: Jünger stellt das Geschehen in übernatürliche, metaphysische Zusammenhänge. Der Krieg ist heilig. Sein Erlebnis entspricht einer religiösen Erfahrung, sein Ablauf einer sakralen Zeremonie. Das Schlachtfeld wird zu einem Wallfahrtsort, zu dem man pilgert, um voller „Ehrfurcht“ (1), ,den Segen über sich ergehen zu lassen“ (156), eine „Prophezeiung“ (163) entgegenzunehmen, an einem Gottesdienst teilzunehmen oder ein Eremitendasein zu führen. Die zentralen (christlich) theologischen Kategorien, die Jünger einsetzt, sind Hölle und Teufel, Wunder, Engel und das „Jenseits“. Da es keine ernsthaften Referenzen auf einen ,Gott‘ gibt, die über das Niveau von Redensarten hinausgingen, verbleibt dies im Metaphorischen.

(15) Ritual: Die einem religiösen Weltbild entsprechende Handlung ist das Ritual. Der Krieg wird zu einer kultischen Veranstaltung. Wenn er ein Opferritus ist, dann sind die Soldaten die Geopferten, und die Offiziere fungieren als Opferpriester.

(16) Fest: Eine vom religiösen Kult sich ablösende Form ist das Fest: Als „blutiges Fest“ (178) bewahrt es das Tödliche des Ritus, als „Fastnacht der Hölle“ (52) die christliche Dimension. Als Orgie erhält es antike, sinnliche und sexuelle Konnotate.

(17) Theater: Jünger fasst den Krieg als „Schauspiel“, als „Spektakel“. Genauer definiert er dieses, generisch, als „Drama“ (25) oder als Tragödie mit Akten, Szenen, 


\section{Lubrich, O. - Sprachbilder des Krieges}

Zwischenspiel, Kulissen und Vorhang. Eine besondere Rolle spielt die Maske: als Maske des Krieges, maskenhafte Gesichter, Totenmaske - oder auch Gasmaske. In einer selbstreflexiven Passage stellt Jünger sein Verfahren der Theatralisierung in Frage: „Heute schmeckt es mir etwas nach Theater“ (101).

(18) Musik: Jünger beschreibt die Geräusche der Schlacht - einschließlich der Laute, die Soldaten von sich geben, als eine Musik, die in verschiedenen Tonarten, Tönen, Klängen und Gesängen zu hören ist, mit Melodie, Takt, Refrain und Ouvertüre, dirigiert von den Kommandeuren der Artillerie. Vier Instrumente treten einzeln hevror: Pfeifen, Trommeln, Glocken - und eine Leier.

(19) Tanz: Wenn Armeen für Musik sorgen, wird das Schlachtfeld zur Tanzfläche: zum „Tanzplatz des Todes“ (166). Als die Beschießung anfing, schreibt Jünger, „begann [...] der Tanz“ (39). Im Krieg scheint alles zu tanzen: Beschuss, Feuer, Splitter, Erde, panische Menschen, Tote, Kompassnadeln und Gegenstände in der erhitzten Luft.

(20) Literatur: Neben dem theatralischen Genre des Dramas bzw. der Tragödie und ästhetischen Kategorien, die auch in der Literatur eine Rolle spielen, wie zum Beispiel das Groteske, wird auch das genuin Literarische, nämlich das Prosaische des Krieges markiert. Einzelne Prosa-Gattungen erhalten eine besondere Funktion: die Fabel, das Märchen, die Schauergeschichte, die Abenteuer-Erzählung und der Wildwest-Roman. Die Handlung des Krieges tendiert ins Fabel- oder Märchenhafte. Die Ästhetik der Gothic Novel wird besonders intensiviert: Das Geschehen ist gespenstisch, unheimlich, „geheimnisvoll“, phantastisch, ein Phantom, ein Spuk voller „Schattengestalten“. Das Abenteuer und das Motiv der Schatzsuche werden mehrfach angesprochen. Auch der Wilde Westen, dessen literarische Rezeption über Karl May (36) eingespielt wird, eignet sich als Deutungsmuster: Der „Wildwest“ (25) ist ein Raum, in dem das Recht des Stärkeren gilt, in dem Freund und Feind als „Cowboy“ (162) und „Indianer[]“ (6) in sprichwörtlicher Gegnerschaft aufeinander treffen. (Figuren wie ,[B]iwak[]“(57) und ,[M]arte[r]“ (143) fügen sich in dieses Bezugssystem.)

(21) Bildende Kunst: Jünger verwendet Kategorien der Ästhetik, um die „Künstleraugen“ (169) eines Grabenkämpfers auf die Stellung der Feinde zu bezeichnen, das ,geniale[]“ (69) Zerstörungstalent und die „genial konstruierten Höllenmaschinen“ (72) - oder auch den ,groteske[n]“ (135) Anblick der Verheerung. Neben den an- 


\section{Lubrich, O. - Sprachbilder des Krieges}

deren Künsten (Theater, Musik, Tanz, Literatur) ist auch die Bildende Kunst metaphorisch ausgeprägt. Das Motiv der Bildhauerei bezieht sich auf die Soldaten, die bereits in der Ausbildung wie eine Skulptur „,nach allen Regeln der Kunst zum militärischen Menschen geschliffen“ (8) oder spätestens im Krieg in eine Plastik gegossen werden. Der Mensch kann aber auch dadurch zur Statue werden, dass er im Angesicht des Grauens wie durch den Blick der Gorgo „versteinert[]“ (124). Die Munition wiederum nimmt den Charakter von Kunstgegenständen an: „Die überall verstreuten Geschosse und Granatsplitter waren von einer schönen, grünen Patina überzogen.“ (43)

(22) Malerei: Der Krieg erscheint als Gegenstand ästhetischer Erfahrung. Im privilegierten Modus optischer Wahrnehmung lässt er sich wie ein Gemälde betrachten. „Die Landschaft war malerisch verziert durch die vielen [...] Kriegsleute“ (9). „Das Bild des Krieges war nüchtern, grau und rot seine Farben“(V). Immer wieder beschreibt Jünger das Geschehen als „Bild“, dessen Ausschnitte gerahmt wirken.

(23) Architektur: Überall treten architektonische Elemente zutage: Die Effekte der Geschosse zeigen sich als Türme, Säulen, Sperren, Riegel, Ketten, Wände, Wölbungen, Bogen oder Brunnen. Aber auch die Haltung der Soldaten (als Pfeiler, Riegel oder Kette), das Aussehen der Stellungen (als Terrasse oder Hütte), die Natur des Schlachtfeldes (als Teppich) und der Eindruck des Schrecklichen werden in der gleichen Bildlichkeit dargestellt.

(24) Sport: Sportarten aus zwei Bereichen werden aufgerufen: der Ballsport, demzufolge die Geschosse und vor allem Handgranaten die Rolle von Bällen einnehmen, und der Kampfsport: Ringen, Boxen und Fechten. Jünger beschwört einen „Sportsgeist“, dessen Ethos den Kampf reguliert.

(25) Spiel: Diverse Spiele dienen als Modelle des Verstehens: Strategiespiele wie Schach, Glücksspiele wie Kartenspiel, Würfeln oder Lotterie, Schießspiele wie Kinderpistolen mit Platzpatronen, Sportschießen auf Zielscheiben und Schießbuden auf dem Rummelplatz. Eine weitere Gruppe bilden Schneeballwerfen, Rasseln, Kegel und andere Kinderspiele und Spielzeuge. Im Hinblick auf eine Offensive, an der er teilnahm, sagt Jünger, dass im Rückblick selbst „die größten der überstandenen Schlachten dagegen ein Kinderspiel schienen“ (143). Demgemäß erfahren sich Soldaten bisweilen als Minderjährige: in der Schlacht fühlen sie sich wie ein Kind, das sich verlaufen hat; 


\section{Lubrich, O. - Sprachbilder des Krieges}

in der Trauer um getötete Kameraden weinen sie wie Kinder; und im Tod nehmen sie das Aussehen von Kindern an.

(26) Schule: Die Idee der Schule durchzieht den Text leitmotivisch: Der erste Kriegsmonat war „eine gute Schule“ (7). Die Straße eines Ortes, in der Offiziere untergebracht sind, „nahm [...] das Aussehen eines Studentenviertels an“ (91). Kampfeseifer wirkt wie pennälerhafte „Rauflust“ (131). Wie einem aufsässigen Primaner hat Jünger einem Gegner die Leviten gelesen, „den frechen Posten durch Handgranaten gezüchtigt“ (88). „Im Kriege lernt man gründlich, aber das Lehrgeld ist teuer.“ (142)

(27) Ziviles Leben: Elemente bürgerlicher Alltagskultur sind über den Text verstreut: Das Kriegsgebiet wird zu einem Jahrmarkt, erstürmte Unterstände zu einem Trödelladen und besetzte Dörfer zu Irrenhäusern. Der Krieg hat dem Gelände seinen „Stempel aufgedrückt“ (28). Ein Unterstand „tropfte“ „,wie eine Gießkanne“ (28) - oder er wird „wie eine Streichholzschachtel zermalmt“ (69). Kampfhandlungen erinnern an Fegen und Wischen, Kämmen und Rasieren, Besuch und Empfang. Einen Offizier bezeichnet Jünger als „unbehagliche[n] Nachbar[n]“ (30). Splitter nehmen die Form von Nadeln an. Eine verkohlte Uniform sieht aus wie ein Frack. Drastisch beschreibt Jünger die Körper der Verwundeten und Toten in Metaphern von Haushaltsutensilien: „Dem einen war der Kopf abgeschlagen[,] und der Hals saß am Rumpf wie ein großer, blutiger Schwamm.“ (76) „Ein anderer, dem ein dreieckiger Lappen vom Hinterschädel herabhing, stieß fortwährend schrille, erschütternde Schreie aus.“(15)

\section{Viertes Feld: Personifizierung}

Über Anthropomorphismen erhält der Krieg menschenähnliche Züge.

(28) Person: Krieg und Tod erscheinen als Figuren, als Allegorien: als finsteres Wesen, Dämon, Moloch oder Monster, das erwartungsvoll zwischen den Armeen steht, um die Menschen in seine Arme laufen zu lassen, das mahnende Rufe ausstößt oder düster murrt, das einen Schatten wirft, seine Fratze zeigt und sich grinsend über das Sterben freut. Sie treten in Erscheinung als Reiter, Jäger oder Unhold (mit Keule). Das Ungeheuer ist nicht nur abstrakt allegorisiert, sondern auch konkret bildlich vorstellbar: mit Krallen, als feuerspeiender Drache, der auf der Lauer liegt, um sich seine Opfer zu 


\section{Lubrich, O. - Sprachbilder des Krieges}

greifen, seinen Atem herüber wehen lässt und die Menschen verschlingt. Sobald die Vorstellung eines überdimensionalen mythischen Unholds installiert ist, werden die Effekte der Schlacht inszenierbar als deren Hieb, Streich, Schlagen, Schmettern, Stampfen, Stapfen, Packen, Stoßen, Hacken oder Blasen. Analog wird der Krieg begreiflich als Kampf zweier Personen, zu denen zusammengefügt die beiden Armeen sich denken lassen: Die gegnerische Seite wird als der Feind gefasst; und auch die deutsche Armee wird zu einer Person stilisiert, die „,auf ständigem Kriegsfuße“ (66) ist und ihre Hand bzw. Faust zum Einsatz bringt. Einzelne Einheiten oder Trupps werden als Kollektivkörper imaginiert, denen beispielsweise „der Mund durch einige Dutzend Schrapnells gestopft“" wird (163). Selbst konkrete Details werden personifiziert: Panzer erscheinen als „Kolosse“ (165). Granaten und Geschosse blinzeln, gurgeln, spucken, toben, werfen, greifen, fassen, erschlagen. Sogar das Gelände wird anthropomorph beschrieben, wenn Kiesgruben und Unterstände gähnen. Alles ringsum nimmt monströse Züge an. „Ihre Tage verbrachten sie in den Eingeweiden der Erde“(VI).

(29) Essen: Der Krieg ist ein Vorgang der Ernährung, ein gigantisches Gelage: Fressen und Gefressenwerden. Die Soldaten haben „Blutdurst“. Sie fressen sich vor und kosten das Schreckliche. Dabei werden sie selbst zu einer Art Nahrung: Sie liegen auf einem Teller (auch die Stahlhelme der Gegner erinnern an Teller) und werden verschlungen. Wie in einem Ofen oder brodelnden Kessel wird Unheilvolles zubereitet.

(30) Sexualität: Jüngers Kriegserfahrung hat eine sexuelle Struktur. Durch die Mobilisierung und seine erste Kampferfahrung wird ihm gewissermaßen die Unschuld genommen. Eine Offensive oder ein Stoßtruppunternehmen haben die Dramaturgie eines Geschlechtsaktes: von leidenschaftlicher Erwartung über Vorspiel, Vorstoß und Eindringen in die feindlichen Stellungen bis zum Abklingen der Erregung nach dem Ende der Kampfhandlungen. Die Sequenz der Schlacht als Sexualakt beschreibt Jünger in den Begriffen Wollust, Lust, „Stellung“, „Höhepunkt“ und Befriedigung. Sein „erste[s] Mal“ hat für den 19-Jährigen eine besondere Bedeutung. Nach einem Tag der Ruhe fühlt er sich „[w]ie neugeboren“ (17). Der Krieg wird zu einer erotischen Erfahrung, das Schlachtfeld zu einem Ort sexueller Begegnung: zu einem „Rendezvous-Platz der Kampftruppen“ (73). Verwundungen sind (mit kitschiger Phantasie) als Pfeile Amors lesbar - oder auch als Penetrationen. „Die Bluse herunterreißend, sah ich, daß ich einen Schuß quer über dem Herzen bekommen hatte.“ (159) Ein Gefreiter [sic!] 


\section{Lubrich, O. - Sprachbilder des Krieges}

namens Hengstmann [sic!] wird zu Jüngers Retter, indem er ihn in seine Arme nimmt und wie der Ritter seine damsel in distress zu den eigenen Reihen trägt, wobei er selbst von einem Schuss getroffen wird: „Hengstmann sank ganz sanft unter mir zusammen.“ (180) Eine weitere Textur provoziert eine homoerotische Deutung des Verhaltens zu den eigenen Leuten: Das Miteinander der Kameraden ist nicht nur als „,[u]nser Verkehr“ (8), sondern auch als partnerschaftliches Zusammenleben lesbar: „Wir [...] führten gemeinsame Wirtschaft.“ (8) Dabei kommt es zu intimen Annäherungen: „Ich schmiegte mich unwillkürlich an einen Mann, der neben mir auf der Pritsche lag.“ (93) Als sie eine andere Einheit in deren Unterkunft stören, kommen diese ,im Negligé herbeigeeilt[]“ (79). Explizite Sexualität findet jedoch nicht statt, „die Erotik fand keinen Raum“ (18). Für die Gespräche der Soldaten gilt ersatzweise um so mehr: „die Erotik spielte eine Hauptrolle“ (136). Leibhaftige Frauen spielen dagegen kaum eine allerhöchstens als Krankenschwestern. Und gerade dann lösen sie bei dem unerfahrenen Frontsoldaten eine Beunruhigung aus, über die Klaus Theweleit einiges zu sagen hätte: „Trotzdem ich das Gegenteil eines Weiberfeindes bin, irritierte mich jedesmal das weibliche Wesen, wenn mich das Schicksal der Schlacht in das Bett eines Krankensaales geworfen hatte. Aus dem männlichen, zielbewussten und zweckmäßigen Handeln des Krieges tauchte man in eine Atmosphäre undefinierbarer Ausstrahlungen. Eine wohltuende Ausnahme bildete die abgeklärte Sachlichkeit der katholischen Ordensschwestern.“(181)

(31) Gespräch: Der Krieg scheint den Regeln der Kommunikation zu folgen, wie ein Gespräch. Die Kanonen sprechen und treten in einen Dialog, in dessen Verlauf gesendet und erwidert wird. Eine Beschießung ist als Gruß zu verstehen. „Manchmal schwieg das Artilleriefeuer“(167).

(32) Cyborg: In Stahlgewittern entwirft eine militärische Anthropologie. „Die menschliche Natur" (103) ist vom Krieg gezeichnet. Der einzelne Soldat ebenso wie die Einheiten werden als technoide Organismen, als Cyborgs imaginiert. Rückgrat, Knochen und Fäuste sind nicht biologisch, sondern aus Eisen. Menschen funktionieren wie Automaten, Maschinen. Die Schlacht vollzieht sich zwischen teils organischen, teils technischen Figuren, „Zwischen zwei ehernen Kräften“(167). 


\section{Lubrich, O. - Sprachbilder des Krieges}

Insgesamt ergibt sich folgende Textur metaphorischer Felder und Codes:

\begin{tabular}{|l|l|l|l|}
\hline NATUR & PRAXIS & KULTUR & PERSON \\
(1) Kosmos & (6) Jagd & (14) Religion & (28) Subjekt \\
(2) Wetter & (7) Viehzucht & (15) Ritual & (29) Essen \\
(3) See & (8) Ackerbau & (16) Fest & (30) Sex \\
(4) Land & (9) Handwerk & (17) Theater & (31) Gespräch \\
(5) Tiere & (10) Bergbau & (19) Musik & (32) Cyborg \\
& (11) Industrie & (20) Literatur & \\
& (12) Arbeit & (21) Bildende Kunst & \\
& (13) Ökonomie & (22) Malerei & \\
& & (23) Architektur & \\
& & (24) Sport & \\
& & (25) Spiel & \\
& & (26) Schule & \\
& & (27) Ziviles Leben & \\
\end{tabular}

\section{Semantik}

In Stahlgewittern lässt sich in (mindestens) 32 metaphorische Codes zerlegen. Die Verbildlichungen sind als einander ablösende, ergänzende und variierende Interpretamente zu verstehen. Was bedeuten diese jedes für sich?

Als astronomisches, meteorologisches, nautisches oder geologisches Phänomen scheint der Krieg den Naturgesetzen zu folgen und sich menschlichem Einfluss zu entziehen. Er kommt den Betroffenen vor wie ein Ausbruch der Elemente. Als Naturereignis wäre er unausweichlich. Und er wäre nicht mit menschlichen Maßstäben bewertbar. Ist diese Naturalisierung als ideologisches Verfahren zu kritisieren (KAEMPFER 1981: 68f.)? Soll der Vorgang als naturgegeben gerechtfertigt und die Verantwortung delegiert werden? Ist Krieg natürlich (Vgl. PONCET 1996)? Äußert sich in der Metapher der Flut eine zeittypische reaktionäre Furcht vor der Auflösung hierarchischer Ordnungen (Vgl. THEWELEIT [1977] 1995)? Oder wäre „,[d]ie ,Naturalisierung“ des Krieges [...] nicht vorschnell kritisch [...] als Ideologisierung abzutun“, da sie immerhin ein 


\section{Lubrich, O. - Sprachbilder des Krieges}

Modell bildet, Distanz schafft und das „erkennende[] Subjekt“ gegen den Gegenstand positioniert (KNEBEL 1991: 392)?

Auch in der Tierwelt verbleibt der Krieg in einem nicht moralisch sanktionierbaren Bereich. In animalischen Akteuren wird er aber zumindest personalisiert und insofern in eine symbolisch deutbare Analogie zu menschlichem Verhalten gebracht. Die Interpretation dieser Analogie ist ambivalent: Obwohl er den Begriff der Fabel selbst einnspielt, hat Jüngers Text kein fabula docet zu bieten. Einerseits ist der Kampf im Tierreich mit den Darwinschen Prinzipien des struggle for life und survival of the fittest assoziiert. Jüngers zoologischer Blick wäre in diesem Sinne sozialdarwinistisch zu verstehen. Allerdings ist die Tierbildlichkeit weder rassistisch codiert, so dass edle Tiere gegen niedere kämpfen würden, noch aristokratisch, weil die Stoßtruppkämpfer zwar als „Tiger“ des Grabens erscheinen, jedoch im nächsten Atemzug als Ameisen beschrieben werden, die vor einer Granate fliehen, oder als Maulwürfe, die sich in der Erde vergraben. Überdies ist die Vertierung, die der Krieg mit sich bringt, das Animalische, das er im Menschen hervorruft, durchaus kritisch lesbar: als ,Antikriegs'-Metaphorik.

Als Jagd, Tierzucht und Ackerbau wird der Krieg in die Sphäre des menschlichen Handelns integriert. Jagd und Tierzucht befinden sich zwischen der zoologischnatürlichen und der menschlich-technischen Welt. Beide implizieren Gewalt: Der Mensch tötet das Tier bei der Jagd oder durch Schlachtung. Insofern auch in diesen Bildern der Mensch als Tier fungieren kan, als gejagtes oder wie Vieh gehaltenes und geschlachtetes, setzt sich jener Aspekt der tierweltlichen Bildlichkeit hier fort. ${ }^{7}$ Als Landwirtschaft dagegen wird der Krieg zu einem an sich friedlichen Vorgang. Er ist organisiert, produktiv und damit positiv konnotiert. Alle drei Bereiche, Jagd, Viehzucht und Ackerbau, implizieren jedoch, kulturanthropologisch gesehen, jeweils einen Umgang mit Aggression und Gewalt: Das Aggressionspotential, das der Jagdverband auf das Tier lenkt, kann der Tierzüchter bei ritueller Schlachtung, im Opfer, kanalisieren (Vgl. BURKERT [1972] 1997). Eine Ackerbau-Gesellschaft ist darauf angewiesen, Ersatzhandlungen in Form symbolischer Riten zu entwickeln, die ohne Blutvergießen

\footnotetext{
${ }^{7}$ Rolf SCHROERS sieht die Motive der Jagd und des Fallenstellens (ebenso wie die der Reise und des Spiegels) im Kontext der in späteren Schriften entwickelten ,stereoskopischen' Suche nach einem tieferen Sinn hinter den Erscheinungen (SCHROERS 1965: 220-224).
} 


\section{Lubrich, O. - Sprachbilder des Krieges}

eine vergleichbare Ventilation ermöglichen und entsprechende den Zusammenhalts stärken. Gelingt dies nicht, läuft sie Gefahr, einen unkontrollierten Gewaltausbruch zu erleben: einen Krieg (Vgl. GIRARD 1972).

Der Soldat als Techniker ${ }^{8}$ und der Krieg als Arbeit (Vgl. NETT/KLUG 1981) sind Erscheinungsformen der Moderne. An ihnen wird das Leben in einer technischen Welt zum Thema. Jüngers Modernität ist einerseits destruktiv, sofern sie das Alte zerstört, andererseits schafft sie etwas Neues und kann bejaht werden. Aus dieser zynischen Perspektive erscheinen Tod und Leid als notwendige Opfer des Fortschrittsprozesses. In Der Arbeiter (1932) ${ }^{9}$ setzte Ernst Jünger diese Verbildlichung fort, indem er den „Arbeiter“ paradigmatisch mit sozial-utopischer Bedeutung auflud. Hier wird nicht mehr der Soldat zum Arbeiter stilisiert, sondern er wird umgekehrt als eine mögliche Erscheinungsform dieses allgemeinen „Typus“ abgeleitet. Das Motiv der Arbeit wird totalitär. Vor dieser Ausweitung jedoch bedeutet die Professionalisierung des Soldatenberufs als $J o b$ gegenüber dem nationalstaatlichen Volksheer eine Milderung des tendenziell totalitären Nationalismus.

Jünger nimmt keine wertende Unterscheidung zwischen verschiedenen Formen des Wirtschaftens vor, nach denen etwa Landwirtschaft, Bergbau und Industrie positiv (schöpferisch) wären, während der Handel und vor allem das Finanzwesen als negativ (nicht schöpferisch) abgewertet würden - wie dies im antisemitischen Diskurs der Fall war (Vgl. AdORNO/HORKHEIMER [1944] 1988). Der Krieg steht selbstverständlich in einem realen Zusammenhang mit der Wirtschaft der kriegführenden Länder. Durch seine Bildlichkeit verweist Jünger auf einen tatsächlichen Zusammenhang. Später wird er den Krieg im Konzept der „Totalen Mobilmachung“ zum Paradigma einer totalisierten Gesellschaftsform ausdeuten. ${ }^{10}$ Auf der anderen Seite macht er seinen ökonomischen Aspekt sichtbar. Kapitalismus ist letztlich ein kriegerisches Handeln; der Krieg die Fortsetzung der Wirtschaft mit anderen Mitteln. In diesem Sinne befindet sich Jüngers Deutung im Einklang mit der Kriegs- und Imperialismustheorie von Marx und Engels bzw. Lenin.

\footnotetext{
8 Zur Typologie von ,Soldat', ,Arbeiter‘, ,Tyrann', ,Ästhet' und ,Anarch` in Jüngers Gesamtwerk vergleiche MERLIO 1995, vor allem 419 ff., und MERLIO 1996.

${ }^{9}$ Ernst JÜNGER, Der Arbeiter [1932], Sämtliche Werke 8, S. 9-317.

${ }^{10}$ Ernst JÜNGER, Die totale Mobilmachung [1931], Sämtliche Werke 7, S. 121-141.
} 


\section{Lubrich, O. - Sprachbilder des Krieges}

Indem man seinen metaphysischen Charakter behauptet, wird der Krieg menschlicher Verantwortung erneut entzogen. Die Möglichkeit seiner rationalen Interpretation ist in Zweifel gestellt. Wenn er gottgewollt ist, erscheint er ebenso unausweichlich wie als Naturereignis. Er besitzt einen tieferen Sinn. Allerdings kann auch dieser Gestus als verständliche Reaktion des Frontsoldaten nachvollzogen werden: Hermann KNEBEL sieht die „konnotative Verbindung mit metaphysischen Konzepten“ als den Versuch an, „die Minimalisierung des Subjekts durch die industrielle Maximierung der technischen Kriegswirkungen“ aufzuheben (KNEBEL 1991: 392).

Das rituelle Opfer ist kollektive Gewalt. Gegenüber dem Mord, dem Pogrom oder dem Überfall handelt es sich jedoch um eine sakral legitimierte, kanalisierte und der Gemeinschaft dienliche Gewalt, die als Ausgangspunkt kultureller Entwicklung verstanden werden kann (Vgl. BURKERT [1966] 1991; LORENZ [1963] 1983). Indem er sich der Opfer-Metapher bedient, unterstellt Jünger eine komplexe Sinnhaftigkeit, eine psychische, soziale und zivilisatorische Zweckmäßigkeit, die dem Krieg in der Realität kaum zukommt. Aber er postuliert immerhin eine Einfügung in Konventionen. Ein Opfer ist kein gewöhnlicher Mord.

Als Fest dagegen wäre der Krieg etwas Angenehmes. Wie das Opfer liegt er außerhalb der Alltagswirklichkeit, in einem symbolisch demarkierten Sonderbereich. Als künstlerische Performanz wird er zu einer uneigentlichen Handlung - auch wenn das Theater in seinem Beginn die Erinnerung an den Opferritus bewahrt haben mag. Sie ermöglicht es dem Betrachter, sich außerhalb der Handlung, nicht auf der Bühne, sondern im „Foyer des Todes“ zu verorten (KRULL 1995: 30f.). Das Motiv von der Welt als Theater, wie es in mittelalterlichen Mysterienspielen, bei Shakespeare (,All the world's a stage...“[SHAKESPEARE [ca. 1599] 1975: II.vii.139]) oder in Calderóns El gran teatro del mundo (CALDERÓN DE LA BARCA [1649] 1987) auftaucht, fügte sich zu einer komplexen Allegorie: Wenn die Welt eine Bühne und das Leben auf ihr ein Schauspiel ist, dann kommt ,Gott ${ }^{`}$ die Funktion des Autors, des Regisseurs und des Kritikers zu, der nach dem Tod, wenn der Vorhang gefallen ist, beurteilt, wie gut die Menschen ihre Rollen gespielt haben, wie konform sie sich zur religiösen Textvorlage verhalten haben, und der ihnen danach ihre Position außerhalb des Theaters, im eigentlichen, ,ewigen“ Leben zuweist. Ein vergleichbar übergeordneter Sinn ist bei Jünger nicht zu erkennen. 


\section{Lubrich, O. - Sprachbilder des Krieges}

Musikalität suggeriert Harmlosigkeit und perfekte Planbarkeit. Jüngers TanzMetapher las Harro Segeberg vor dem Hintergrund von Kleists Essay Über das Marionetten-Theater als Umschlagen von Disziplin und Technik in Immaterialität und Grazie: als Perfektion des mechanisierten Menschen (SEGEBERG 1991: 356 f.).

Indem Jünger seinem Text durch zahlreiche Zitate eine zusätzliche Ebene verleiht, kennzeichnet er ihn nicht nur als Kriegsliteratur, sondern als moderne Literatur, insofern diese sich durch gesteigerte Selbstreferentialität und Selbstreflexion auszeichnet. Indem er den Konstruktcharakter seiner Schrift markiert, wirkt er nicht nur einer ästhetischen, sondern auch einer politischen Ideologie entgegen, die er in anderer Hinsicht zu befördern scheint. Selbstreferentialität ist tendenziell anti-autoritär.

Die Vorstellung vom Soldaten als Skulptur nimmt sowohl das monumentale Heldengedenken wie andererseits auch, indem das darzustellende Objekt als grauenvoll beschrieben wird, die Erinnerungskultur der Antikriegs-Denkmäler vorweg: Arno Breker und Käthe Kollwitz zugleich. Das Provozierende der Malerei-Motivik liegt darin, dass Jünger die unbeteiligte Position des distanzierten Betrachters reklamiert, deren Interesselosigkeit dem Grauen Hohn zu sprechen scheint. In der Erzählliteratur der Moderne werden katastrophische Kriegsbilder indes regelmäßig als apokalyptische Diagnosen ekphrastisch, in Form Gewalt darstellender Gemälde, repräsentiert (Vgl. BUCH 2002). Gerade die evidentia des Bildes erlaubt es, das Grauen wirksam mitzuteilen. Jüngers Bilder bleiben trotz aller Ästhetisierung Bilder des Schreckens.

Die Architektur ist das konstruktive Metier par excellence. Der Krieg ist das destruktive Ereignis schlechthin. Die Unangemessenheit dieser Metaphorik ist offenkundig. Allerdings hat sie einen diskursiven Index: Christoph Asendorf situiert die architektonische Visualisierung von Geschossbahnen ${ }^{11}$ im Kontext avantgardistischer Vorstellungen von Design, Urbanistik, Anthropologie und Technologie, die im Motiv der Stromlinie, Parabel oder Flugbahn mit dem Militärischen zusammenfielen. ${ }^{12}$

\footnotetext{
${ }^{11}$ Asendorf bezieht sich auf eine Stelle in der siebenten Fassung (S. 166): „Unsere Lage war nun so, daß wir unter der Feuerglocke saßen wie unter einem enggeflochtenen Korb.“

12 ASENDORF 1993. Neben Ernst Jünger stehen u.a. Le Corbusier und Peter Behrens im Fokus der Untersuchung.
} 


\section{Lubrich, O. - Sprachbilder des Krieges}

Als theatralisches, musikalisches, tänzerisches, bildnerisches literarisches oder architektonisches Phänomen besitzt der Krieg einen ästhetischen Wert. ${ }^{13}$ Ernst Jünger wurde immer wieder mit dem Ästhetizismus und insbesondere mit Friedrich Nietzsche in Verbindung gebracht (Vgl. KAEMPFER 1984; OHANA 1989). Walter Benjamin stellte im Nachwort zur dritten Fassung seines ,Kunstwerk-Aufsatzes‘ zwei allgemeine Thesen auf, erstens: der Faschismus laufe ,auf eine Ästhetisierung des politischen Lebens hinaus“; und zweitens: „Alle Bemühungen um die Ästhetisierung der Politik gipfeln in einem Punkt. Dieser eine Punkt ist der Krieg.“ (BENJAMIN [1936-1939] 1977: 506; Vgl. HILLACH 1979). In einer Rezension kommt er direkt auf Ernst Jünger zu sprechen und unterstellt ihm genau dies: ,eine hemmungslose Übertragung der Thesen des L'Art pour l'Art auf den Krieg.“" ${ }^{14}$ Andererseits sind Jüngers Verfahren der Ästhetisierung, wie Karl Heinz BOHRER gezeigt hat, seine „Ästhetik des Schreckens“, in der dem ,Augenblick` gesteigerter Wahrnehmungsschärfe eine besondere Bedeutung zukommt, auch modernistisch und nicht affirmativ, sondern zeitdiagnostisch lesbar. ${ }^{15}$

Das Motiv des Sports impliziert, dass der Krieg harmlos sei, aber auch, dass er nach den Regeln der fairness ablaufe. Dieser Gedanke ist regulativ zu verstehen. Wenn der Krieg ein Sport ist und die Soldaten die Sportler, dann muss es ein Regelwerk geben: ein Kriegsrecht. Indem Jünger dies prinzipiell fordert und anhand eigener Erlebnisse demonstriert, hält er an einer völkerrechtlichen Regulierung fest. Er widerspricht der nationalistischen Doktrin, dass dem Feind mit Hass zu begegnen, und der Ludendorffschen Theorie, dass der Krieg „total“ zu führen sei.

Auch als Spiel wäre der Krieg etwas Harmloses und Angenehmes. Ulrich Prill sieht bei Jünger generell eine „ludische Einstellung“ (PRILL 2002: 33). Indem sich das Motiv des Spiels mit dem des Kindes verbindet, eröffnet sich indes wiederum eine

\footnotetext{
13 Zum ästhetizistischen Dandy-Motiv: Ernst JÜNGER, Sturm [1923], Sämtliche Werke 15, S. 11-74; vergleiche hierzu PLARD 1968.

${ }^{14}$ BENJAMIN [1930] 1991: 240. Benjamin kritisiert eine technologische Verschärfung des Idealismus und gebraucht dabei eine Bildlichkeit, die bei Jünger nicht vorkommt: „so weit man über den Grabenrand blicken konnte, war alles Umliegende zum Gelände des deutschen Idealismus selbst geworden, jeder Granattrichter ein Problem, jeder Drahtverhau eine Antinomie, jeder Stachel eine Definition, jede Explosion eine Setzung, und der Himmel darüber bei Tag die kosmische Innenseite des Stahlhelms, bei Nacht das sittliche Gesetz über dir. Mit Feuerbändern und Laufgräben hat die Technik die heroischen Züge im Antlitz des deutschen Idealismus nachziehen wollen. Sie hat geirrt.“(BENJAMIN [1930] 1991: 247)

15 BOHRER 1978, vor allem 138-403. Zur Kritik an Bohrers modernistischer Jünger-Interpretation: HEMMERICH 1983.
} 


\section{Lubrich, O. - Sprachbilder des Krieges}

kritische Bedeutung: Der Krieg ist wie die Handlung von Kindern: vorrational, egoistisch, grausam. Ihn als Schule zu bezeichnen, wirkt einerseits verharmlosend. Andererseits spezifiziert Jünger nicht, was es denn sei, das der Soldat lerne. Er lernt nämlich unter Umständen gerade dies: dass der Krieg schrecklich und verbrecherisch ist.

Eine solche Ambivalenz ist in den übrigen Metaphern aus dem zivilen Leben nicht angelegt. Josef Peter STERN hat die Grausamkeit von Jüngers „Embattled Style“ als Manierismus kritisiert und das umgekehrte Übergreifen einer militärischen Metaphorik auf zivile Bereiche in Der Arbeiter beschrieben: ,a piece of prose is not a ,military exercise', nor do ,guns speak', except by way of a worn-out metaphor.“ (STERN 1978: 119, 225 [Fußnoten]).

Als Person wird der Krieg zu einem Subjekt - und damit bewertbar. Da Jünger ihn monströs zeichnet, ist er kaum positiv zu verstehen. Die Soldaten müssen ihm gegenüber eine Haltung einnehmen: Angst, Ekel, Ablehnung. Als Nahrungsaufnahme verstanden, wäre der Krieg auf mehrdeutige Weise notwendig: Das Leben und das Sterben im Schützengraben werden zu Etappen der Nahrungskette und des Kreislaufs der Materie. Das ist als Verklärung, als Vulgärdarwinismus - aber auch als Zynismus lesbar.

Wenn der Kampf sexuell ist, dann ist er triebhaft, lustvoll und abermals notwendig. Da sich diese Sexualität unter Männern abspielt, ist sie (implizit oder explizit) homoerotisch. Sie kann als Überwindung von Differenzen verstanden werden (Vgl. SEGEBERG 1991: 347). Das erotische Verhältnis zum Feind läuft auf sadomasochistische Perversion hinaus - aber auch auf eine gewisse Gleichsetzung und Verbindung. Wenn der Krieg als Gespräch inszeniert wird, als zivilisierte Form der Interaktion, dann ist dies so haarsträubend, dass sich ein weiteres Mal die Frage stellt: Ist das provozierend naiv, verharmlosend und ideologisch oder ironisch und subversiv zu verstehen? Desavouiert die Metaphorisierung des Krieges durch dessen Antithese, kommunikatives Handeln, das eine oder das andere?

Das Cyborg-Motiv ist nicht per se reaktionär. Matthew BIRO unterscheidet progressive und regressive Varianten aus der Weimarer Zeit (BIRO 1994, vor allem 97110). Jünger steht für die Befürwortung einer Technologie, die den Menschen autoritär verwandelt; im Gegensatz zur kritischen Inszenierung bei Raoul Hausmann und zur 


\section{Lubrich, O. - Sprachbilder des Krieges}

ambivalenten und selbstreflexiven bei Fritz Lang. In Stahlgewittern verbindet das Motiv mit einer Schärfung der Sinne, Die Totale Mobilmachung mit der technologischen Transformation der Welt und Der Arbeiter mit der Epiphanie des neuen Menschen im kybernetischen Staat. ${ }^{16}$

Indem Jünger den Krieg naturalisiert, pragmatisiert, kulturalisiert und personifiziert, macht er ihn deutbar. Jede Metapher besitzt einen gemeinsamen Nenner mit ihrem Objekt, ein tertium, das die Übertragung ermöglicht. Durch ihre Unschärfen und Sinnüberschüsse erzeugt sie allerdings Bedeutungen, die problematisch sind. Die metaphorischen Interpretationen sind in ihrer ideologischen Dimension zu befragen. Ihre Implikationen sind unterschiedlich: Verharmlosung und Entstellung, Fatalisierung und Verantwortung, Verherrlichung und Subversion. Die Metaphern enthalten Ambivalenzen, ein zynisches, ironisches oder kritisches Potential. ${ }^{17}$ Jüngers Verfahren sind alles andere als eindeutig. ${ }^{18}$

Es besteht die Versuchung, den späteren Ernst Jünger in diesen allerersten Text ,hinein` zu lesen und die erste Fassung von In Stahlgewittern vor dem Hintergrund der folgenden Fassungen und der übrigen Schriften zu sehen. ${ }^{19}$ Die Kontroverse um den Autor überschattet die Lektüre des Werkes. ${ }^{20}$ Ein Mythos der Person erschwert eine

\footnotetext{
${ }^{16}$ Vergleiche auch PEKAR 2000; zur Technisierung der Natur: MOTTEL 2000.

${ }^{17}$ Hans Verboven hat, indem er die Metapher als Ideologie betrachtet und vor den ,metaphorischen Gefahren der Sprache“ warnt, die These entfaltet, Jüngers „Sprache und Denken“ seien von der „Struktur“ ihrer Metaphern derart ,geprägt“, dass diese ,,in ihrer Gesamtheit [...] eine in literarische Form gegossene Ideologie oder Philosophie des Krieges“, ,eine in der literarischen Sprache verkörperte Ideologie“, „eine Legitimation des Krieges und [...] somit eine klare ethische (ideologische) Wahl" darstellten, die als in sich kohärent zu betrachten wäre; dass Jünger mithin ,,in seinen Metaphernmodellen gefangen“ sei (VERBOVEN 2003: 245-252). Im Unterschied zu diesem Ansatz soll hier der Vorschlag gemacht werden, den Widersprüchen innerhalb der Jüngerschen Metaphorik nachzugehen.

18 Roger Woods sah Symptome psychischer und politischer Beunruhigung in Spannungen zwischen euphorischer Erwartungshaltung und Desillusion, fieberhafter Bedeutungssuche und Sinnlosigkeit, soldatischem Gemeinschaftsgefühl und Isolation, rauschhaftem Kampf und Zweifeln an dessen militärischer Führung: „One overlooked aspect of Jünger's portrayal of the First World War is the range of attitudes (often irreconcilable) which underly his thinking." (WOODS 1990: 80)

${ }^{19}$ Eva Dempewolf hat die Verschiebungen zwischen den Fassungen historisch im Hinblick auf Jüngers biographische Situationen und politische Intentionen untersucht und dabei auch die Veränderungen diverser Bildlichkeiten betrachtet (DEMPEWOLF 1992: 13, 30, 191, 194, insbesondere 78-160). Hans Verboven hat nicht nur einen ,Fassungsvergleich' erarbeitet (VERBOVEN 2003: 253-262), sondern die Entwicklung der Kriegsmetaphorik durch Ernst Jüngers gesamtes Frühwerk hindurch rekonstruiert und theoretisch konzeptualisiert (als Ausbau, Steigerung, Stillstand und Regression, Metaphorisierung formaler Vergleiche oder Ausweitung der Metaphorik auf nicht-kriegerische Bereiche).
}

${ }^{20}$ Zur Kontroverse um Jünger: MORENO CLAROS 1995. - Für eine liberale Rezeption: LEHNER 1995. 


\section{Lubrich, O. - Sprachbilder des Krieges}

unvoreingenommene Rezeption. ${ }^{21}$ Dabei bietet der Text von 1920 die Möglichkeit, nicht den fertigen Kriegsverherrlicher und Faschisten wiederzufinden (Vgl. BERNHARD 1963), sondern dessen Genese zu beobachten. Die nationalistischen und totalitären Positionen sind hier nicht voll entwickelt. Es sind Ansätze erkennnbar, die später entfaltet wurden. Es zeigen sich aber auch alternative Potentiale, die entweder nicht ausgebaut oder wieder verdrängt und zum Teil später reaktiviert wurden.

\section{Funktion}

An die Frage nach den Bedeutungen der Bildlichkeiten schließen sich weitere Fragen an: 1. Lassen sich Typen von Metaphern unterscheiden? 2. Welchen Gesamteindruck erzeugen die Sprachbilder? 3. In welchem Verhältnis stehen sie zueinander? Gibt es eine übergeordnete Systematik? Geben die Tropen dem Kriegsgeschehen insgesamt eine Interpretation? 4. Berühren oder überschneiden sie sich? 5. Ist eine Entwicklung zu beobachten? 6. Gibt es eine Logik, nach der sie auf die Kriegsparteien verteilt sind? 7. Wie verhalten sich die Metaphern zu nicht-metaphorischen Verwendungen des gleichen Wortmaterials? 8. Wird die Metaphorisierung auf einer Meta-Ebene reflektiert? 9. Welche Metaphern tauchen im Text auf, ohne dass sie weiter entwickelt würden? Und welche werden, bezeichnenderweise, nicht eingesetzt? 10. Wie angemessen sind die von Jünger gewählten Bilder? 11. Wie tritt ihr Erkenntniswert zutage? 12. Und bedient sich Jünger einer (proto-) faschistischen bzw. -nationalsozialistischen Sprache?

(1) Typen: Jüngers Sprachbilder sind, formal betrachtet, unterschiedlicher Art: Nach dem Grad ihrer Ausdrücklichkeit lassen sich verschiedene Typen unterscheiden: erstens reflektierte Bilder (Vergleiche, die durch metatextuelle Formulierungen als solche eingepasst oder anmoderiert sind); zweitens explizite Metaphern (die als solche erkennbar sind, weil ihre Metaphorizität deutlich ausgeprägt ist und die Bildlichkeit in der Lektüre nicht von der pragmatischen Bedeutung des Wortes absorbiert wird); und drittens implizite Metaphern (deren ,erste` Bedeutung, die buchstäbliche, den metaphorischen Aspekt verdeckt, so dass er sich erst bei gezielter Beobachtung und im

\footnotetext{
21 „Sein größtes Kunstwerk war er selber.“ (BURGER 1998: 447)
} 


\section{Lubrich, O. - Sprachbilder des Krieges}

Zusammenspiel mit gleichartigen Bildern erschließt). Es ist nicht möglich, diese Typen trennscharf zu unterscheiden, da sie ineinander übergehen.

(2) Dichte: Alle drei Typen scheinen in Jüngers Text mit weit größerer Frequenz aufzutauchen, als dies in literarischen Texten durchschnittlich der Fall ist. Der Gesamteindruck ist zunächst rein quantitativ der einer ungeheuren Dichte, einer metaphorischen Wucherung. Dieser Befund lässt sich als Symptom verstehen: Die außerordentliche Alterität der Erfahrung führt zu einer Variation von Deutungsversuchen, die sich implizit - und möglicherweise zum Teil unbewusst - in einer Vielzahl sprachlicher Bilder äußert. Deren Pluralität zeugt von der Unmöglichkeit, den Gegenstand nicht nur begrifflich, sondern überhaupt gedanklich zu bewältigen, in ein schlüssiges Modell zu bringen und sprachlich darzustellen. ${ }^{22}$

(3) Relation: Wie verhalten sich die Bereiche, in denen sich die Metaphern bewegen, zueinander? Sie ergänzen sich - synchron - als Ebenen der Sublimation: vom Einfachen (Wetter, Ackerbau, Ritual, Essen) zum Komplexen (Tiere, Ökonomie, Literatur, Dialog). Des Weiteren ergeben sich - diachron - Zusammenhänge, deren Logik die einer impliziten Sozial-, Wirtschafts- und Kulturgeschichte zu sein scheint: sozial und wirtschaftlich versteht Jünger den Krieg als Jagd, Viehzucht, Ackerbau, Handwerk, Bergbau, Industrie, Finanz- und Handels-Ökonomie; kulturell deutet er ihn ebenfalls auf verschiedenen Entwicklungsstufen: als Ritual, Fest, Sport, Theater, Musik, Tanz, Bildhauerei, Malerei, Architektur und Literatur.

(4) Interferenz: Wie verhalten sich die Bilder und Bedeutungen zueinander? Es ergeben sich mehr oder weniger komplizierte Kombinationen, deren Elemente häufig nicht kompatibel sind - beispielsweise Motive der See und des Tanzes („Die Sturmwelle tanzte wie eine Reihe von Gespenstern durch weiße, wallende Dämpfe.“ [146]), der Landschaft und der Architektur (,durch einen Wald kirchturmhoher Granatfontainen“ [97]), der Tierwelt und des Gartenbaus (,Erde sprang auf in fauchenden Fontänen“ [105]). In einem einzelnen Wort wie „Garbe“ überlagern sich mehrere Bedeutungsschichten: die Vorstellung des Getreidebundes aus der Landwirtschaft, die über-

\footnotetext{
22 Auf inhaltlicher Ebene beschrieb Hans-Harald Müller, wie Jüngers Versuch, sein Kriegserlebnis individualistisch und heroisch zu deuten, in Konflikt geriet mit der Notwendigkeit, ein entindividualisiertes Geschehen zu beschreiben (MÜLLER 1995, insbesondere 18-24); dies ,führte Jünger [...] bis an die Grenze der Einsicht, daß die diesem Erlebnis zugrunde liegenden Motive und Gefühle nicht kommunizierbar sind.“ (MÜLLER 1995: 23)
} 


\section{Lubrich, O. - Sprachbilder des Krieges}

tragen wurde auf die Flammen eines Feuerwerks; die ästhetischen Konzepte der Haltung, des Schmucks und des Gewandes (garb); und die Bezeichnung von Fleischstücken aus der Metzgerei. ${ }^{23}$ Der Krieg wird mit Bedeutungen befrachtet, die einander überlagern und miteinander in Konflikt geraten. Die Metaphorik wird potenziert und an den Rand ihrer Funktionsfähigkeit getrieben.

(5) Variation: Wie verteilen sich die Metaphern? Eine quantitative Analyse könnte die Gewichtungen zeigen, indem sie die statistische Häufigkeitsverteilung der einzelnen Bilder untersucht. Wichtiger ist jedoch die qualitative Frage: Was wird wann und wo wie metaphorisiert? Auffällig ist eine Häufung auf den ersten beiden Seiten des Vorworts und auf der ersten Seite des Haupttextes, wo die Motive eingeführt werden. Eine Abnahme auf Grund eines Gewöhnungseffektes tritt nicht ein. Aber die Frequenz variiert: Sie wird immer dann dichter, wenn von Kampfhandlungen die Rede ist, während sie wieder abnimmt, sobald Aufenthalte hinter der Front beschrieben werden. Die Intensität der Metaphorik indiziert die Intensität des Grauens. Bemerkenswert ist ein Moment, in dem die Metaphorisierung zeitweise aussetzt: Zu Beginn des Kapitels „Die große Schlacht“, einem der Höhepunkte des Textes, beschreibt Jünger, wie seine Kompanie, die sich in einem Trichter für den Angriff sammelt, von einer Granate getroffen wird (140 f.). Die Wirkung des Geschosses ist verheerend: Von mehr als 150 Mann entgehen nur 63 dem Tod oder einer schweren Verwundung. Leutnant Jünger reagiert, indem er „aufsprang und planlos in die Nacht rannte.“ (141) Dann erleidet er einen Zusammenbruch: „Ich warf mich zu Boden und brach in ein krampfhaftes Schluchzen aus, während die Leute düster um mich herumstanden.“(141) Und er deutet an, dass dieses Erlebnis für ihn traumatisch sein würde: „Solche Augenblicke vergißt man nie.“ (141) Es handelt sich um das Szenario eines shell shock. Bezeichnenderweise spielen die üblichen Sprachbilder genau hier keine Rolle. Die Kette der Tropen wird unterbrochen. In Panik ist es nicht mehr möglich, Metaphern zu bilden.

(6) Dekonstruktion: Jeder Krieg beruht auf einem binären Freund-Feind-Verhältnis. Einerseits hat es bei Jünger den Anschein, als würden die Gegner regelmäßig mit Metaphern belegt, die pejorativer Natur sind. So erscheinen zum Beispiel die Engländer als „Schweine“ $(22,159)$. Andererseits ist eine stereotype Tiermetapher, mit der

\footnotetext{
${ }^{23}$ Deutsches Wörterbuch von Jacob und Wilhelm Grimm, Leipzig 1878. Eintrag „Garbe“.
} 


\section{Lubrich, O. - Sprachbilder des Krieges}

Jünger sich und seine Kameraden belegt, die des „Frontschweins“ (28, 93). Ausdrücklich wendet er die Bedeutung des Wortes ,Schwein“ als ,Schurke‘ auf beide Seiten an: „,Il y a des cochons aussi chez vous! „, (28) ruft ihm ein englischer Offizier zu, mit dem er sich über das Niemandsland hinweg unterhält, als ein deutscher Soldat einen inoffiziellen Waffenstillstand gebrochen hat. Diesen Satz macht sich Jünger später zu eigen: „Il y a des cochons partout.“ (62) Umgekehrt dreht er das englische Schimpfwort, „"the Huns', (153), auf die Gegner zurück - und nimmt ihm die abwertende Bedeutung, wenn er von „hünenhafte[n] Engländer[n]“" spricht (179). ${ }^{24}$ Indem dieselben Bilder auf beide Seiten angewandt werden, setzen sie die Opposition außer Kraft, die sie zu bestätigen schienen. Der Gegensatz, der den Krieg motiviert, löst sich auf.

(7) Referentialität: Die Metapher wird dadurch noch weiter geführt, dass diese „Schweine“, die deutschen Soldaten, es nicht nur mit anderen, englischen, zu tun haben, sondern auch mit realen, von denen sie sich ernähren: sie essen „Schweinefleisch“ [93]. Die Metaphern kollidieren mit ihren realen Entsprechungen. Der Effekt ist ein gewisses Flimmern, eine Irritation zwischen literarischer Trope und außerliterarischer Realität.

Dieser Effekt scheint von Jünger gesucht zu werden. Für fast alle Bereiche, denen er seine Figurationen entnimmt, schafft er reale pendants. So zum Beispiel eine „Mühle“ (45), ein „Bergwerk“ (19, 43), ein „Kalkwerk“ (das ausgerechnet „Chez-bontemps“ heißt, 78), die „Walze“ eines Grammophons (154), „das Eisengerüst einer zerstörten Zuckerfabrik“ (135), eine „Kapelle“ (165), ein photographisches „Bild“ (147) und einen „Rahmen Patronen“ (150), eine wirkliche Unterhaltung mit den Feinden (131) und eine höchst reale „Riesengestalt [...], eine weiße Keule schwingend“ (85). Es verschwimmt die Grenze zwischen der Allegorie des Krieges und den aus ihr in die Wirklichkeit übertragenen Vorstellungen. Die Soldaten feiern, wenn sie sich betäuben, ein „Trankopfer“ (79). Die Abteilungen „strömten“ aus den Kneipen. (8) Tatsächlich schneidet jemand Draht „mit seiner Zigarrenschere“ (46). Die Soldaten machen nicht nur metaphorisch, sondern auch wirklich Musik. Jünger selbst „trommelte mit den Füßen zur Erwärmung gegen die Stollenrahmen“ (135). „Weihnachtslieder“, werden „mit MG.‘s übertönt““ (29). Analog werden schlafende Soldaten „durch [...] Mückenschwärme, Granaten [...] gestört“ (97). Jünger beschreibt sich nicht nur, er verstellt sich

\footnotetext{
${ }^{24}$ Das Wort „Hüne“ leitet sich her von „Hunne“ (lat. Hunnus).
} 


\section{Lubrich, O. - Sprachbilder des Krieges}

auch, vorsichtshalber (in der Dunkelheit), als Tier: „Ich [...] gab [...] eine Reihe von

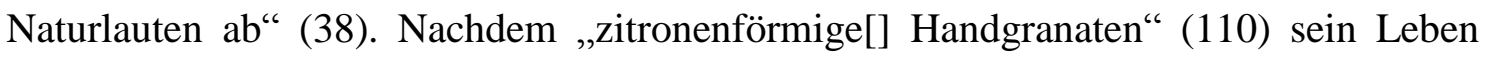
bedroht haben, erhält der Verwundete „Zitronenlimonade“ (181).

Der Begriff der Schule durchzieht den Text in vielfältigen Wendungen, figurativen und buchstäblichen: Schulen im Kriegsgebiet, Schulungen für die Soldaten, Schüler und Lehrer sowie Schul-Erinnerungen. Das Spiel hält Einzug, indem Soldaten zwischendurch tatsächlich, zum Beispiel Skat, spielen; aber auch, indem zwischen den Stellungen ,in grausigem Kontrast ein Kinderspielzeug“ (19) liegt. Jüngers Stoßtrupp bedient sich als Transportmittel zurückgelassener Kinderwagen (73). Und schließlich leben im Kampfgebiet sogar Kinder: „Ahnungslose Kinder spielten“ (18). Die verharmlosenden Bilder werden mit der traurigen Wirklichkeit konfrontiert.

Besonders skurril ist die mehrfache Bedeutung der Maske. Neben die Maske des Krieges, die maskenhaften Gesichter, die Totenmaske und die Maske der Schauspieler tritt die Gasmaske. Wenn von „Masken“ die Rede ist, muss der Leser ihre Bedeutung aus dem Kontext erschließen. Zugleich ist das Motiv selbstreflexiv: Es verweist auf Jüngers Verfahren, die Realität bildlich zu maskieren. „Ich setzte die Maske auf“ (42).

Im Bereich der Natur wird deutlich, wie sich metaphorische und buchstäbliche Bedeutungen überlagern - und wie beide wiederum mit anderen Bedeutungen derselben Wörter in Konflikt geraten. Naheliegenderweise sind es Phänomene des Wetters, die den Bildern, durch die der Krieg inszeniert wird, entsprechen: Regen, Schnee, Sturm und Sonnenschein. Bisweilen berühren sich beide, die reale und die metaphorische Dimension: „Ein Gewitter zog auf, seine Donnerschläge wurden übertönt von dem einsetzenden Lärm eines neuen Trommelfeuers.“ (107) Es ist nicht mehr eindeutig zu entscheiden, wie die Wörter primär zu lesen seien; beispielsweise wenn vom „Frühlingserwachen“ (137) die Rede ist, während einerseits tatsächlich der Frühling beginnt und andererseits der deutsche Großangriff losbricht. Darüber hinaus werden Namen zitiert, die mit denselben Bildern arbeiten: So gibt es das „Kalkwerk 'Chez-bons-temps““(78) das unter schwerem Beschuss steht. Auf einer anderen Ebene bewegen sich alternative Verwendungen derselben Vokabeln. Beispielsweise wird das Wort Regen als „Regen von Blumen“ (1) gebraucht, Hagel als „Hagel von Schimpfworten“ (163), Schauer als „Todesschauer“ (52), Donner als Ausruf „'Donnerwetter!‘,, (40) und Blitze als „,blitzschnelle[], logische[] Schärfe“ (36). 


\section{Lubrich, O. - Sprachbilder des Krieges}

Die nautische Metaphorik scheint dadurch beglaubigt $\mathrm{zu}$ werden, dass tatsächlich ein „Schiffsgeschütz“ (45) zum Einsatz kommt; dass Soldaten im durchnässten Gelände beinahe ,im Schlamm [...] ertrunken“ (121) wären; dass „ein schmutziger Strom mein Sitzloch gurgelnd bis obenhin füllte“ (103); und dass Jünger beim Graben versteinerte „Muscheln, Seesterne[]“ (108) findet.

Verschiedene Ebenen der Jagd werden gespiegelt: Real ist die Rede von einem Befehl zur „Schonung der französischen Jagd“ (125). Die Regiments-Reserve ist in einem Forsthaus untergebracht, das „'Fasanerie“,, (3) getauft wird. Burleske Variante sind die „Läusejagd“ (9) und die „Rattenjagd“ (38). Einzelne Requisiten stellen einen link zwischen realer und metaphorischer Dimension her: Ein Soldat schreitet, „eine lange grüne Jägerpfeife im Munde, mit umgehängter Flinte durch das Maschinengewehrfeuer, als ob es zur Hasenjagd ginge.“ (152) Im Militärjargon gibt es die Waffengattung der ,Jäger“ $(151,152)$.

Reale Tiere tauchen auf: ein Maulwurf, Ratten und Mäuse, Fliegen, Läuse, Regenwürmer, Enten und Wasserhühner, Fische, ein Hecht, ein Schäferhund, ein Terrier, Hasen und Rebhühner. Es gibt Eier und einen Ziegenstall. „Schnecken und Maulwürfe lagen“ nach einem Gasangriff „,tot umher“ (43). Jünger gibt sich als Käferkundler zu erkennen: als „Entomologe“ (108). Bestimmte Tiere scheint er - wie die Geschosse, die sie im übrigen verkörpern - als lästig zu empfinden: „Eine Lerche stieg hoch und ärgerte uns durch ihr Trillern.“ (86) Was ihn irritiert, scheint der Widerspruch zu sein, der „Kontrast“ zwischen dieser realen Erscheinung und der militärischen Bedeutung, die er ihr zugewiesen hat: „Eine Lerche steigt hoch; ich empfinde ihr Getriller als aufdringlichen Kontrast, es irritiert mich.“ (22)

Die Bildlichkeit wird ins Tatsächliche eingelöst: Reale Schulen, Spiele und Spielzeuge, Mühlen, Bergwerke und Fabriken, Masken und Lieder, Naturphänomene, Jagden und Tiere lösen Jüngers Metaphern von den Rändern her auf, so dass sie ihre Figuralität in Frage stellen; und sie stören ihre Funktion, indem sie ihre Unangemessenheit sichtbar machen.

(8) Un/Angemessenheit: Sind die Sprachbilder, die Jünger verwendet, ihrem Gegenstand angemessen? Das Gegenteil scheint auf der Hand zu liegen. Selbstverständlich ist der Krieg weder ein Konzert noch ein Gebäude und schon gar kein Gespräch. Der verharmlosende Kontrast wirkt schockierend. Er führt das Unfassliche des Krieges 


\section{Lubrich, O. - Sprachbilder des Krieges}

und die Unfähigkeit der Sprache, ihn zur Darstellung zu bringen, vor Augen. In Stahlgewittern ist der angestrengte Versuch, dem Krieg durch Verbildlichung einen Sinn abzugewinnen; und zugleich, indem die Haltlosigkeit dieses Versuchs erkennbar wird, das Eingeständnis von dessen Scheitern. Gert Mattenklott sprach von Jüngers Arbeiter im Blick auf Nietzsche als einem ,tragischen Typus, der auf Fiktionen angewiesen ist, um das Leben aushalten zu können.“ (MATTENKLOTT 1999: 30). Dieser Satz lässt sich an Jüngers erstem Buch variieren: Ernst Jünger ist auf Metaphern angewiesen, damit er das Grauen des Krieges ertragen kann. Die Metaphern haben eine Schutzfunktion. Je intensiver das Grauen, desto dichter die Bildfrequenz.

(9) Ansätze \& Alternativen: Außerhalb der Motivketten und Bildfelder gibt es einzelne Metaphern, die in keine Netzwerkstruktur eingebunden sind. Diese Ansätze zeigen, welche möglichen Bedeutungsräume nicht erschlossen werden, welche denkbaren Interpretationen im Hintergrund bleiben. Da ist zum Beispiel das Motiv der Hinrichtung („Henkersmahlzeit“ [110]), demzufolge der Krieg als massenhafte Exekution durch eine tyrannische Obrigkeit hätte verstanden werden können; oder das des Mordes („Mordwaffen“ [131], „Mordinstrumente“ [164]), nach dem er als Massenmord, als Verbrechen anzusehen wäre; des weiteren die Krankheit bzw. Medizin („Pest[]“ [VI], „Kugelspritzen“ [158]), die es erlauben könnte, ihn als epidemische Krise der Vernunft zu konzeptualisieren; Peitsche und Fesseln (,aufgepeitscht“ [157], „fesselte“ [178]), die ihn als Sadomasochismus darstellen ließen. Darüber hinaus gibt es isolierte, stumpfe Metaphern - Bildlichkeiten, die zum Teil an anderer Stelle weiter exploriert werden: beispielsweise die „Strahlung“, die in späteren Texten eingeführt wird (Vgl. PORATH 1995: 252 f.; HÜPPAUF 1997: S. 22), die Elektrizität („Gegenpole[]“ [167]), ${ }^{25}$ die Archäologie oder der Alptraum. Signifikant ist des Weiteren, welche Bildlichkeiten überhaupt nicht eingesetzt werden, obwohl sie sich in vorhandene Felder einfügen ließen: zum Beispiel die der Sintflut, die eine apokalyptische Vision ergeben hätte; ${ }^{26}$ oder die Marionette (die in Der Kampf als inneres Erlebnis entwickelt wird), die das Idiotische des militärischen Gehorsams versinnbildlichen könnte.

\footnotetext{
${ }^{25}$ Helmut Lethen beobachtet, wie Jünger im Arbeiter eine neue Bildlichkeit einführt: ,er wechselt die technische Zentralmetapher. Jünger ist einer der ersten Schriftsteller, der in einer Gesellschaftsanalyse das Modell des elektrischen Schaltkreises ins Zentrum rückt.“ (LETHEN 1994: 209).

${ }^{26}$ Als apokalyptische Interpretation: GERHARDS 1999, vor allem 74-123.
} 


\section{Lubrich, O. - Sprachbilder des Krieges}

(10) Selbstreferenz: Jünger praktiziert eine militärische Metaphernbildung nicht nur, sondern er thematisiert sie auch als sprachliche Praxis im Krieg. Anhand diverser Begriffe reflektiert er die Wucherung figurativer Bezeichnungen, indem er sie in Anführungszeichen setzt, als „sogenannte“ markiert oder ihr Zustandekommen erläutert: Unterstände heißen „'Tropfsteinhöhle““ (5) oder „'Zum Männerbad““ (5), das Dörrgemüse der Feldküche „'Drahtverhau““ (5) oder „'Flurschaden““ (5), eine Ruine mit Ausblick über die Front „'Bellevue““ (19), der Sitz des Kommandeurs „'Mäuseburg““ (93), ,ein zerschossenes [...] Haus [...], das seinen Namen wahrscheinlich wohl verdiente“, „Rattenburg“ (97) und ein „Sanitätsunterstand „Kolumbusei“, (96). Kampfgas nennen die Soldaten „Offensiv-Parfüm“ (162), Panzersperren „Spanische[] Reiter“ (111), bestimmte Handgranaten „Eier-Handgranaten“ (111), einen Sprengsatz „Waschkorb-Minen“ (40), einen anderen „Flaschenminen“ (171). Die Kugelfänge sind „sogenannte Siegfriedbleche“ (163). Und selbst der improvisierte Tisch wird zu einem „Sogenannten Tisch“ (163). „Tanks [...] trugen zum Teil spöttische, drohende oder glückbringende Namen und Kriegsbemalungen“ (165). „Für dieses Brodeln entfernten Kanonendonners hatten wir den klangvollen Frontausdruck ,Es wummert' geprägt.“ (174) Soldaten haben an einem Schützengraben „ein Schild mit der halbverwischten Aufschrift ,Drachenweg“, (127) angebracht. Einzelne Frontabschnitte erhalten konnotationsreiche Namen - zum Beispiel „,[d]as wüste Gelände, das wir ,Die Walachei“ getauft hatten“ (162). Ein ,Kollege“ hat „,seinen lächerlichen kleinen Unterstand“ nach einer Romanfigur „'Villa Leberecht Hühnchen“ getauft“" (38). Schlafen bezeichnet man als „'röcheln“, wie der Fachausdruck lautet“" (22) (während in Jüngers Prosa umgekehrt das Röcheln eines Sterbenden dem Schnarchen eines Schlafenden gleichkommt: „Sein schnarchendes Röcheln“ [157]). Schließlich 'markiert' Jünger sogar die Rhetorik der Befehle und Berichte: ,wie die schöne Befehlsformel lautet“ (178).

An allen diesen Stellen verweist er auf eine kriegsübliche Praxis: die Ausbildung eines soldatischen Jargons; dabei distanziert er sich von diesem Jargon, indem er ihn in Anführungszeichen setzt, um ihn durch eine eigene Bildsprache zu ersetzen. Diese Distanzierung einer literarischen von einer trivialen Figuration macht einen der Unterschiede aus zwischen In Stahlgewittern und einer konventionellen Frontkämpferliteratur, wie sie in den zwanziger Jahren massenhaft publiziert wurde. 


\section{Lubrich, O. - Sprachbilder des Krieges}

Während er soldatische Terminologie aus gewisser Distanz betrachtet, installiert Jünger, wie gesehen, seine eigene Metaphorik auf verschiedenen Ebenen: als Bilder des Krieges (primäre metaphorische Ebene), alternative Bilder aus demselben Wortmaterial (sekundäre Ebene), Eigennamen (zwischen Zeichen und Realität) und entsprechende Realien (dargestellte Wirklichkeit).

(11) Epistemologie: Ernst Jüngers Text impliziert eine epistemologische Fragestellung (und er expliziert sie auch ansatzweise): Welche Funktion haben Sprachbilder bei der Aneignung der Wirklichkeit? Wie prägt figurative Sprache unser Wahrnehmen, Erkennen, Verstehen und Denken? Inwiefern ist unsere Wirklichkeit durch Tropen konstruiert? Welche kommunikative Funktion haben diese? Haben Sie den heuristischen Wert provisorischer Modellbildung $?^{27}$ Und welche weiteren Funktionen besitzen sie? Welchen existentiellen, psychologischen Mechanismen folgt die Metaphernbildung? Indem In Stahlgewittern diese Fragen aufwirft, schafft das Buch eine reflexive Distanz zu seiner poetischen Praxis.

(12) Faschismus: Ist Ernst Jüngers Sprache faschistisch? Russell Berman definierte Ekphrasis, die Privilegierung bildlichen Sehens über die Schrift, als Strategie faschistischer Repräsentation (an Leni Riefenstahls Triumph des Willens und Jüngers Arbeiter). Seine These ist ,the descriptive rhetoric of fascist representation“ (BERMAN 1989: 64; vgl. RAUSCH 1995; BOEHM 1995). Ist Jüngers Bildgebung per se faschistisch? Angemessener scheint eine konkretere Frage zu sein: Welche der Jüngerschen Bildlichkeiten, oder genauer: welche ihrer Elemente und Ausformungen, entsprechen einer nationalsozialistischen Imagination? Das Bild des Tigers, zum Beispiel, tauchte im Zweiten Weltkrieg in der Benennung eines Kampfpanzers auf; das Motiv des Rittertums in der Selbstinszenierung der SS; die Idee des Menschenopfers wurde nach der Niederlage von Stalingrad propagandistisch genutzt; der nihilistische Todeskult bildete ein zentrales Element der Weltanschauung. ${ }^{28}$ Einige Bildlichkeiten, deren sich Jünger in seiner Kriegsbeschreibung bediente, hatten ein unheilvolles Nach-

\footnotetext{
27 Trifft bereits auf das Erstlingswerk zu, was von den Texten der dreißiger und vierziger Jahre gesagt werden kann, nämlich dass Jüngers Verfahren einem „Symbole und Zeichen gleichsam ,durchwandernden“ Meditieren“" gleichkomme? (SADER 1996: 223)

28 ,Jüngers metaphorischer Archaismus, der fossilische Charakter seiner Bildersprache berühren sich mit einer Tendenz, die zu den Grundtendenzen des europäischen Faschismus gehören dürfte.“ (KAEMPFER 1976: 149)
} 


\section{Lubrich, O. - Sprachbilder des Krieges}

leben. ${ }^{29}$ Sie verfestigten sich zu ideologischen Konzepten (,Totale Mobilmachung', ,Arbeiter', ,Inneres Erlebnis'). Historisch lassen sich im Bewältigungsversuch des Ersten Weltkrieges Entwicklungslinien des Faschismus in ihrem Entstehen betrachten. Ernst Jüngers Sprache ist partiell und potentiell faschistisch.

Die einfachste Methode, dem Krieg einen ,Sinn` zuzuweisen, ist die vordergründig ideologische: seine Rechtfertigung durch eine übergeordnete Instanz (Gott, Führer, Kaiser, Volk, Nation, Staat, Armee, Befehl) oder ein abstraktes Prinzip (Patriotismus, Freiheit, Glaube, Fortschritt, Frieden). Ernst Jüngers Texte sind insofern aufschlussreich, als sie auf derartige explizite Deutungen weitgehend verzichten. Statt dessen ist die indirekte Strategie erkennbar, den Krieg, das radikal ,Andere' des zivilisierten Lebens, mit den Mitteln der Sprache zu verarbeiten: in einer poetischen Ökonomie der multiplen Figuration. Bereits der Titel zeigt diese Technik kumulativer Verbildlichung an: Stahl + Gewitter. Die tiefe Irritation, die der Krieg auslöst, scheint nach einem Modus literarischer Deutung zu verlangen, der nicht inhaltlich explizit, sondern formal implizit und damit flexibler und subtiler ist. Die Metaphorisierung dient der Rationalisierung und Sinnstiftung. Der Krieg wird übersetzt und in Zusammenhänge eingebettet, in denen er als scheinbar ,normale‘ Erfahrung nachvollziehbar wird.

Durch seine exzessiven, konkurrierenden Metaphorisierungen nimmt Jüngers Text eine Vielzahl divergenter Wertungen vor. Er spricht dem Krieg einerseits tiefere, positive Bedeutungen zu, die er andererseits wieder in Frage stellt. In Stahlgewittern ist eher ein Kaleidoskop unterschiedlicher Deutungsversuche als eine autoritäre Verherrlichung. Der Text handelt von der Unbegreiflichkeit eher als von der Herrlichkeit des Krieges. In seiner Widersprüchlichkeit ist das Buch in zweifacher Weise als Dokument lesbar: einerseits erlaubt es, eine Faszination zu rekonstruieren und ein ideologisches Denken in seinen Ursprüngen zu beobachten, das später in den Nationalsozialismus einmünden wird; andererseits bietet es die Gelegenheit, die Schrecken des Krieges in den Versuchen reflektiert zu sehen, sprachlich mit ihnen umzugehen.

\footnotetext{
${ }^{29}$ Kunicki beobachtet, beispielsweise, eine „Hervorhebung des Landsknechtmäßigen“ von der ersten zur zweiten Fassung und dessen „Schwund“ von der dritten zur vierten (KUNICKI 1993: 54 f., 156). - Jüngers Kriegsdarstellung verändert sich zwischen den Weltkriegen beträchtlich. Ernst KELLER (1994) erkannte einen Wandel (um 1934) von Ästhetisierung und Apologie zu Selbstreflexion und Kritik. Jan Philipp Reemtsma analysiert Jüngers „moralische Verstörung“, „Selbstekel“ und „Verleugnungsmechanismus“ anhand von „Auslassungen“ im Kaukasus-Tagebuch als Technik des „Fading“ (REEMTSMA 1998: 320 f.,
} 


\section{Lubrich, O. - Sprachbilder des Krieges}

In der ersten Fassung von In Stahlgewittern hat Ernst Jünger dem Ersten Weltkrieg weniger explizit einen ideologischen Sinn zugeschrieben, als ihn implizit durch eine intensive Bildsprache mit Bedeutungen aufzuladen. Die Jüngerschen Sprachbilder lassen sich in Codes analysieren, nach Typen differenzieren und in ihrer Dichte, Relation, Interferenz und Variation beschreiben. Die implizite Bedeutungszuweisung ist keineswegs einsinnig. Die Metaphern konnotieren abweichende Positionen; sie geraten miteinander in Überschneidung und zueinander in Widersprüche. Sie erzeugen eine widerständige Semantik, die nicht nur als Ausdruck einer ideologischen Haltung, sondern auch als Symptom einer Verunsicherung lesbar ist.

\section{Literaturverzeichnis}

ADORnO, Theodor W. / HORKHEIMER, Max. Elemente des Antisemitismus. Grenzen der Aufklärung. In: ADORNO, Theodor W. / HORKHEIMER, Max. Dialektik der Aufklärung [1944]. Frankfurt am Main, 1988, 177-217.

ASENDORF, Christoph. Totale Mobilmachung / Total Mobilisation. In: Daidalos 47, Wege / Paths, 1993: 90-95.

BENJAMIN, Walter. Das Kunstwerk im Zeitalter seiner technischen Reproduzierbarkeit (Dritte Fassung) [1936-1939]. In: TIEDEMANN, Rolf / SCHWEPPENHÄUSER, Hermann (ed.). Gesammelte Schriften. 7 Bände in 14 Teilbänden, Band 1.2. Frankfurt am Main, 1977, 471-508 („Nachwort“: 506 ff.).

BENJAMIN, Walter. Theorien des deutschen Faschismus. Zu der Sammelschrift Krieg und Krieger. Herausgegeben von Ernst Jünger [1930]. In: TIEDEMANNBARTELS, Hella (ed.). Gesammelte Schriften. Band 3 [Kritiken und Rezensionen]. Frankfurt am Main, 1991, 238-250.

BERMAN, Russell A. Written Right Across Their Faces: Ernst Jünger's Fascist Modernism. In: HUYSSEN, Andreas und BATHRICK, David (ed.). Modernity and the Text. Revisions of German Modernism. New York / Oxford, 1989, 60-80.

BERNHARD, Hans-Joachim. Die apologetische Darstellung des imperialistischen Krieges im Werk Ernst Jüngers. In: Weimarer Beiträge 2, 1963: 321-355.

BIRO, Matthew. The New Man as Cyborg: Figures of Technology in Weimar Visual Culture. In: New German Critique 62, 1994: 71-110.

BLUHM, Lothar. Natur in Ernst Jüngers Tagebüchern aus dem Zweiten Weltkrieg. In: Wirkendes Wort 1, 1987: 24-32.

329 f.). Lothar BLUHM (1987) sieht Jüngers Hinwendung zur Natur (und Kunst) als innere Emigration; ähnlich BLUHM 1995; vergleiche BULLOCK 1992: 89. 


\section{Lubrich, O. - Sprachbilder des Krieges}

BLUHM, Lothar. Ernst Jünger als Tagebuchautor und die ,Innere Immigration'. Gärten und Straßen (1942) und Strahlungen (1949). In: MüLlER, Hans-Harald / SEGEBERG, Harro (ed.). Ernst Jünger im 20. Jahrhundert. München, 1995, 125153.

BlumenBerg, Hans. Paradigmen zu einer Metaphorologie. Frankfurt am Main, 1960.

BLUMENBERG, Hans. Die Lesbarkeit der Welt. Frankfurt am Main, 1981.

BOEHM, Gottfried. Fundamentale Optik. In: FIGAL, Günter / SCHWILK, Heimo (ed.). Magie der Heiterkeit. Stuttgart, 1995, 9-24.

BOHRER, Karl Heinz. Die Ästhetik des Schreckens. München, 1978.

BUCH, Robert. Violent Images. Ekphrastic Writing in Claude Simon and Peter Weiss. Dissertation, Stanford, 2002.

Bullock, Marcus. Fallen Altars Are Occupied by Demons: The Disenchantment of Ernst Jünger. In: GRIMM, Reinhold / HERMAND, Jost (ed.). 1914/1939. German Reflections of the Two World Wars. Madison, 1992, 70-90.

BURGER, Rudolf. Herzstillstand. In: Merkur 590, 1998: 447-449.

BURKERT, Walter. Homo Necans. Interpretation altgriechischer Opferriten und Mythen [1972]. 2. ed. Berlin / New York, 1997, 8-96 („Opfer, Jagd und Totenriten“).

BURKERT, Walter. Griechische Tragödie und Opferritual [1966]. In: BURKERT, Walter. Wilder Ursprung. Opferritual und Mythos bei den Griechen. Berlin, 1991, 1339.

CALDERÓN DE LA BARCA, Pedro. El gran teatro del mundo [1649]. In: FRUTOS CORTÉS, Eugenio (ed.). Pedro Calderón de la Barca. El gran teatro del mundo. El gran mercado del mundo. Madrid, 1987, 39-89.

DE MAN, Paul. Rhetoric of Tropes (Nietzsche). In: DE MAN, Paul. Allegories of Reading. Figural Language in Rousseau, Nietzsche, Rilke, and Proust. New Haven / London, 1979, 103-118.

DEMPEWOLF, Eva. Blut und Tinte. Eine Interpretation der verschiedenen Fassungen von Ernst Jüngers Kriegstagebüchern vor dem politischen Hintergrund der Jahre 1920 bis 1980. Würzburg, 1992.

GERHARDS, Claudia. Apokalypse und Moderne. Alfred Kubins „Die andere Seite“ und Ernst Jüngers Frühwerk. Würzburg, 1999.

GIRARD, René. La violence et le sacré. Paris, 1972, 63-104 („La crise sacrificielle“).

GRIMM, Jacob / GRIMM, Wilhelm. Deutsches Wörterbuch. Leipzig, 1878.

HEMMERICH, Gerd. Ernst Jünger: Ein moderner Autor? In: PESCHEL, Dietmar (ed.). Germanistik in Erlangen. Hundert Jahre nach der Gründung des Deutschen Seminars. Erlangen, 1983, 389-396.

HILlaCH, Ansgar. The Aesthetics of Politics: Walter Benjamin's Theories of German Fascism. In: New German Critique 17, Special Walter Benjamin Issue, 1979: 99-119. 


\section{Lubrich, O. - Sprachbilder des Krieges}

HÜPPAUF, Bernd. Unzeitgemäßes über den Krieg. Ernst Jünger: Strahlungen (1938-48). In: WAGENER, Hans (ed.). Von Böll bis Buchheim: Deutsche Kriegsprosa nach 1945. Amsterdam / Atlanta, 1997, 13-47.

JÜNGER, Ernst. In Stahlgewittern. Aus dem Tagebuch eines Stoßtruppführers. Hannover, 1920. [Weitere Werke Ernst Jüngers werden in den Fußnoten zitiert.]

KAEMPFER, Wolfgang. Das schöne Böse. Zum ästhetischen Verfahren Ernst Jüngers in den Schriften der dreißiger Jahre im Hinblick auf Nietzsche, Sade und Lautréamont. In: Recherches Germaniques 14, 1984: 103-117.

KAEMPFER, Wolfgang. Ernst Jünger. Stuttgart, 1981.

KAEMPFER, Wolfgang. ,Der Tod ist ein Meister aus Deutschland'. Zu einem Leitmotiv im nihilistischen Schrifttum Ernst Jüngers. In: Recherches Germaniques 6, 1976: 136-151.

KELLER, Ernst. Wrestling with an Old Trauma: Ernst Jünger's Changing Perception of Destructiveness. In: AUMLA 81, 1994: 21-31.

KING, John. ,Wann hat dieser Scheißkrieg ein Ende?" Writing and Rewriting the First World War. Schnellroda, 2003.

KNEBEL, Hermann. ,Fassungen': Zu Überlieferungsgeschichte und Werkgenese von Ernst Jüngers In Stahlgewittern. In: SEGEBERG, Harro (ed.). Vom Wert der Arbeit. Zur literarischen Konstitution des Wertkomplexes ,Arbeit' in der deutschen Literatur (1770-1930). Tübingen, 1991, 379-408.

KRULL, Wilhelm. Im Foyer des Todes. Zu Ernst Jüngers In Stahlgewittern und anderen Texten über den Ersten Weltkrieg. In: ARNOLD, Heinz Ludwig (ed.). Ernst Jünger. Göttingen, 1995, 27-35.

KUNICKI, Wojciech. Projektionen des Geschichtlichen. Ernst Jüngers Arbeit an den Fassungen von In Stahlgewittern. Frankfurt am Main u.a., 1993.

LAKOFF, George / JOHnSON, Mark. Metaphors We Live By. Chicago, 1980.

LEHNER, Kurt M. Vom Stahlgewitter zum Anarchen. Ernst Jünger - ein politischer Denker? In: Liberal 2, 1995: 81-87.

LETHEN, Helmut. Verhaltenslehren der Kälte. Lebensversuche zwischen den Kriegen. Frankfurt am Main, 1994, 187-215 (,Jüngers Fall ins Kristall“).

LORENZ, Konrad. Das sogenannte Böse. Zur Naturgeschichte der Aggression [1963]. München, 1983.

LUBRICH, Oliver. Das Schwinden der Differenz. Postkoloniale Poetiken. Bielefeld, 2004, 148- 224. [Dissertation, 2003]

MatTenklott, Gert. Das Neue im Alten: George, Jünger, Benn. In: Der Neue Mensch. Obsessionen des 20. Jahrhunderts. Stuttgart, 1999, 27-35.

MERLIO, Gilbert. Les Figures du pouvoir chez Ernst Jünger. In: DAVID, Claude (ed.). Les Songes de la raison: Mélanges offerts à Dominique Iehl. Bern, 1995, 418434.

MERLIO, Gilbert. Les images du guerrier chez Ernst Jünger. In: BELTRAN-VIDAL, Danièle (ed.). Images d'Ernst Jünger. Bern, 1996, 35-55. 


\section{Lubrich, O. - Sprachbilder des Krieges}

MoRENO Claros, Luis Fernando. Un centenario polémico: Ernst Jünger en Alemania. In: Revista de Occidente 175, 1995: 129-149.

MotTEL, Helmut. Technische Paradiese - Zur poetologischen Funktion der MetaPhorisierung technischer Perfektion im Werk Ernst Jüngers. In: STRACK, Friedrich (ed.). Titan Technik. Ernst und Friedrich Georg Jünger über das technische Zeitalter. Würzburg, 2000, 225-242.

MüLLER, Hans-Harald. ,Im Grunde erlebt jeder seinen eigenen Krieg‘. Zur Bedeutung des Kriegserlebnisses im Frühwerk Ernst Jüngers. In: MÜLLER, Hans-Harald und SEgEBERG, Harro (ed.). Ernst Jünger im 20. Jahrhundert. München, 1995, 13-37.

NeGt, Oskar / KLuge, Alexander. Geschichte und Eigensinn. Frankfurt am Main, 1981, 797-860.

NIETZSCHE, Friedrich. Ueber Wahrheit und Lüge im aussermoralischen Sinne [1873]. In: COLLI, Giorgio und MonTINARI, Mazzino (ed.). Friedrich Nietzsche. Kritische Studienausgabe. 15 Bände, Band 1. Berlin / München, 1988, 873-890.

OHANA, David. Nietzsche and Ernst Jünger: From Nihilism to Totalitarianism. In: History of European Ideas 11, 1989: 751-758.

PEKAR, Thomas. ,Organische Konstruktion“. Ernst Jüngers Idee einer Symbiose von Mensch und Maschine. In: STRACK, Friedrich (ed.). Titan Technik. Ernst und Friedrich Georg Jünger über das technische Zeitalter. Würzburg, 2000, 99-117.

PlARD, Henri. Une œuvre retrouvée d'Ernst Jünger: Sturm (1923). In: Études Germaniques 4 (92), 1968: 600-615.

PONCET, François. La vague, Leitmotiv Jüngerien. In: BELTRAN-VIDAL, Danièle (ed.). Images d'Ernst Jünger. Bern, 1996, 85-104.

PORATH, Erik. Strahlungen Ernst Jüngers. Anmerkungen zu einem Tagebucheintrag. In: Wirkendes Wort 2, 1995: 241-257.

PRILL, Ulrich. „mir ward Alles Spiel“. Ernst Jünger als homo ludens. Würzburg, 2002, 31-48.

RAUSCH, Jürgen. Ernst Jüngers Optik. In: ARBOGAST, Hubert (ed.). Über Ernst Jünger. Stuttgart, 1995, 49-72.

ReEmtsma, Jan Philipp. ,Es schneet der Wind das Ärgste zu'. Ernst Jünger im Kaukasus. In: REEMTSMA, Jan Philipp. Mord am Strand. Allianzen von Zivilisation und Barbarei. Hamburg, 1998, 316-347.

SADER, Jörg. ,Im Bauche des Leviathan'. Tagebuch und Maskerade. Anmerkungen zu Ernst Jüngers Strahlungen (1939-1948). Würzburg, 1996.

SCHROERS, Rolf. Der kontemplative Aktivist. Versuch über Ernst Jünger. In: Merkur 3 (204), 1965, 211-225.

SEGEBERG, Harro. Regressive Modernisierung. In: Vom Wert der Arbeit. Zur literarischen Konstitution des Wertkomplexes "Arbeit" in der deutschen Literatur (1770-1930). Tübingen, 1991, 337-378.

ShaKeSPEARE, William. As You Like It [ca. 1599]. Arden edition. LATHAM, Agnes (ed.). London, 1975, II.vii.139. 


\section{Lubrich, O. - Sprachbilder des Krieges}

STERN, J[osef] P[eter]. The Embattled Style: Ernst Jünger, In Stahlgewittern. In: KLEIN, Holger (ed.). The First World War in Fiction. A Collection of Critical Essays. London / Basingstoke, 1978, 112-125.

THEWELEIT, Klaus. Männerphantasien [1977], 2 Bände. Band 1: Frauen, Fluten, Körper, Geschichte. Band 2: Männerkörper. Zur Psychoanalyse des weißen Terrors. München, 1995.

Verboven, Hans. Die Metapher als Ideologie. Eine kognitiv-semantische Analyse der Kriegsmetaphorik im Frühwerk Ernst Jüngers. Heidelberg, 2003.

Woods, Roger. The Conservative Revolution and the First World War: Literature as Evidence in Historical Explanation. In: The Modern Language Review 1, 1990: 77-91.

Recebido em 30/09/2010

Aprovado em 13/10/2010 